\title{
Chapitre 11 La RDA : prélèvement métadiscursif dans l'extériorité constitutive du dire
}

Quel que soit le souci - parfois aigu - du déjà-dit dans lequel se fait un discours, dont peut témoigner en lui une RDA proliférante, ce qu'il en représente n'est qu'une part de ce qui y joue. C'est au « manque » radical de la RDA au regard de ce qui, illimité, fait la matière même du dire, que tient son pouvoir propre dans le dire - celui d'un prélèvement opéré dans l'ailleurs qui baigne le dire, instituant ces autres spécifiques qu'elle retient, passés par le « filtre » de la conscience métadiscursive, comme partenaires rencontrés par le dire sur son chemin... Mettre en regard, pour un discours, quant à l'extériorité $\boldsymbol{E}$ qui y joue, les traces de présence - que peut notamment y faire apparaître l'analyse de discours, conçue comme pratique réglée de « lecture » dans une mémoire associée - et les formes de représentation $\boldsymbol{E}$, énoncées et observables au fil du dire, éclaire le travail configurateur de la RDA : l'image dont le discours se dote par sa RDA se charge de sens par ce qui, de l'altérité, se trouve, par la sélection qu'elle opère, comme passé sous silence : incorporé, assimilé, ignoré, refoulé, insu...

\section{La représentation $E$ ' envisagée sur fond de la présence $E$ dans quelques discours}

Les discours dits « monologiques » portent à l'extrême la nécessité de penser la fonction de la représentation de l'autre dans son rapport avec la présence de celui-ci : comment, sinon sur le fond des extériorités dont un tel discours se révèle tissé, appréhender dans sa dimension configurante de leurre la fonction de la RDA - dans ce cas par son absence - productrice de l'image d'un discours solitaire, homogène, absolu, du « UN ».

L'étude de la « langue de bois soviétique » par Patrick Sériot est une illustration de cette approche, menée dans une perspective d'AD, au double plan des « traces » et des " marques » de l'interdiscursivité dans le discours : à une présence « constante de discours adverses et antérieurs » ${ }^{1}$ mise au jour systématiquement, notamment dans les nominalisations, forme apte à porter du préconstruit, et dont la surabondance est un trait de ce discours, répond, à la « surface » du dire - là où les formes de représentation dessinent l'image d'un dire « par ses autres » (c'est-à-dire les autres qu'il se reconnaît) - une quasi-absence de formes

1 Sériot (1986b : 30), cf. ci-dessus chap. 10, note 74, p. 401.

ว Open Access. () 2020 Jacqueline Authier-Revuz, published by De Gruyter. (cc)BY-NC-ND This work is licensed under the Creative Commons Attribution-NonCommercial-NoDerivatives 4.0 License. https://doi.org/10.1515/9783110641226-020 
de RDA. Ainsi, dans cette articulation, le plan métadiscursif de la RDA apparaît-il comme opérant une « véritable expulsion [...] de sujets d'énonciation conflictuels en surface assertée » ${ }^{2}$ et la spécificité de la « langue de bois » est-elle saisie comme « une tension extrême entre une homogénéité, un monolithisme déclarés et une hétérogénéité fondamentale. $»^{3}$.

La place du discours autre - présence et représentation - singulièrement celle du discours de l'opposition est l'un des axes privilégiés par Freda Indursky ${ }^{4}$ dans son investigation du discours, au long des 21 années de la dictature militaire au Brésil (1964-1985), de ses « présidents généraux », menée diachroniquement, dans le cadre de l'AD. Dans la première période, au muselage brutal de l'opposition dans les faits - interdite d'expression, mutisée ou emprisonnée... - répond le statut qu'elle reçoit dans le discours de la dictature : celui d'une entité dangereuse, faite de «traîtres » ou " ennemis de la patrie », le plus souvent désignée comme " la subversion, l'anarchie, le désordre, la désagrégation ${ }^{5}$ ", etc., mais en aucune façon comme source d'un discours autre, de contestation, susceptible d'affecter le discours « UN » du pouvoir. A partir des années 70, parallèlement à la progressive transformation du rapport de force desserrant, ébranlant et finalement renversant le pouvoir militaire, le discours de l'opposition va forcer les portes - et l'autisme du discours de la dictature. Net indice, accentué dans les dernières années du cycle militaire, du changement de la conjoncture, l'entrée d'un discours de l'opposition, qu'il n'est plus possible d'annuler, se fait dans l'articulation des deux plans, différenciés, de leur présence $\boldsymbol{E}$ et de leur représentation $\boldsymbol{E}$ ' : à travers une variété de formes de « discours rapporté » $\boldsymbol{E}$ ', le discours « donne la parole » à une opposition reconnue porteuse d'un discours adverse, en se « configurant » lui-même par là, dans ce rapport polémique. Cependant que, sur l'autre plan $\boldsymbol{E}$, la présence, souterraine, de nombreux éléments repris tacitement - consciemment ou non - au discours de l'opposition, par le discours des militaires ${ }^{6}$ atteste de la puissance avec laquelle le discours « montant » du mouvement populaire s'impose à un discours autoritaire fragilisé qu'il investit (« contamine ») de ses manières de dire, ses catégories, ses points de vue. Sur le fond $\boldsymbol{E}$ d'une emprise, sur le discours des militaires,

2 Sériot (1986a : 39).

3 Sériot (1986b : 30).

4 Indursky (1997a), à partir d'une thèse soutenue en 1992, particulièrement la 3 e partie consacrée à « La construction de l'homogénéité imaginaire et ses ruptures ». Menée indépendamment, et dans un autre cadre théorique, la description de Forget (1992) conduit à des observations analogues.

5 Cf. les extraits traduits en français dans Indursky (1997b).

6 Que F. Indursky, soulignant l'entrée incognito dans le fil du discours de ces fragments venus d'ailleurs, appelle « incises discursives ». 
du discours de l'autre, l'image $\boldsymbol{E}$ ', par laquelle, via la RDA, il se configure dans l'image d'un discours menant un débat, sans compromis, avec l'adversaire, revêt quelque chose d'une fonction défensive de dénégation.

C'est, aussi, l'écriture singulière d'un journal d'écrivain que C. Rannoux (2004) - dans son entreprise de caractériser différentiellement, " par leurs autres » représentés, « l'écriture de soi » de plusieurs diaristes - éclaire, de façon aiguë, en montrant comment, chez le Léautaud des années 1939-19427, le tracé d'autres discours, sourcilleusement distingués du sien propre, se dessine, dans un exemplaire aveuglement de l'énonciateur, sur un fond, ignoré, de présence de la discursivité environnante. S'il est, en effet, une image dans laquelle Léautaud aime à se reconnaître c'est, cent fois reformulée, celle d'un esprit lucidement indépendant, à la singularité de « marginal », réfractaire à toute forme d'emprise ou d'affiliation. Traduction méta-énonciative de ce souci de distinction, les si nombreux guillemets qui affectent, curieusement, des termes non pas vraiment « spécialisés » mais présentant l'ombre d'une appartenance à quelque domaine socio-professionnel, tels mise en page, blanc, placard, chapeau (de l'imprimerie), melon (du chapelier), crapaud (du tapissier) ${ }^{8}$ etc. : dépourvus de toute nuance critique, leur valeur est de pure distanciation singularisante, simple occasion pour l'énonciateur, en se démarquant d'un quelconque usage «social », d'afficher sa foncière «non-appartenance ». La même position se retrouve au plan idéologique dans la figure adoptée d'observateur neutre, à distance surplombante des points de vue partisans des autres ${ }^{9}$ - fiction " d'atopie idéologique » en $\boldsymbol{E}$ ', qui ne rencontre pas seulement les limites qu'impose, à la distanciation généralisée d'avec « tous les jargons », le caractère essentiellement « commun » du langage, mais, en $\boldsymbol{E}$, le démenti de l'emprise flagrante du discours pétainiste dans l'expression au jour le jour, par le diariste, de ses goûts, sentiments, opinions « personnelles ».

Ainsi (en $\boldsymbol{E}^{\prime}$ ), la distribution des guillemets sur des mots « pas à soi » rejouet-elle, au plan méta-énonciatif, la position affichée vis à vis des deux camps - favorables ou hostiles à la politique vichyssoise de collaboration : aux guillemets tenant à distance, par exemple, d'un côté les désignations pétainistes des résistants comme « traîtres à la patrie », ou de la République honnie par l'Etat Français instauré par Vichy, comme « l'ancien régime », répondent, de l'autre,

7 «Coupe » dans son immense Journal littéraire - de 1893 à sa mort en 1956 - qui permet la comparaison synchronique avec le Journal de guerre de Malaquais.

8 Voir Rannoux (2004) : chap. 1.3 « Les jargons».

9 Cf. cité in Rannoux (2004) : « Je ne les [les résistants à Londres appelés par certains « traîtres à la patrie »] approuve ni ne les désapprouve. [...] J'écris en spectateur, par esprit critique. (22 juin 1942), et « Je ne suis pas du tout de droite si je ne suis pas de gauche [...]. Je sais fort bien ce que je suis : rien, neutre, indépendant, en marge » (17 septembre 1942). 
ceux qui tiennent à distance, et à chacune de leurs occurrences, « l'occupant », «les occupants » pour désigner la présence allemande ${ }^{10}$. Tandis que (en $\boldsymbol{E}$ ) le discours propre résonne souvent comme un écho direct du discours vichyssois dominant : ainsi, la prise de distance par rapport au mot « d'ancien régime », va-telle de pair avec une évocation « personnelle » des tares d'une République dont il faut se garder de souhaiter le retour qui paraît émaner - sans « filtrage » aucun, dans une parole « ventriloquée » - des rituelles diatribes contre « la Gueuse » des tenants de l'Etat français :

L'excès de désordre, de scandales au grand jour ou cachés, des tendances démagogiques en discours ou en actions, du manque de sens moral chez les écrivains, [...] tout ce qu'on peut penser qui reviendrait [...]. (17 juillet 1942).

De la même façon, c'est, alors même qu'il explicite une réticence à être étiqueté comme antisémite, que le discours trouve «ses » mots - le « faciès indéniable » dans le répertoire de l'antisémitisme le plus avéré :

Je me suis trouvé, en rentrant, dans le métro avec Galtier-Boissière. Il me dit qu'il vient de relire [...] Chroniques dramatiques et qu'il a fait cette remarque que j'y montre déjà un fort antisémitisme. Si on peut appeler antisémitisme au sens courant du mot le fait du déplaisir de voir jouer la Comtesse du Mariage de Figaro par une petite juive au faciès indéniable [...]. (3 décembre 1942).

C'est encore dans cette perspective d'appréhension de l'autre représenté comme tel sur fond d'un ailleurs constitutif « fait sien » que F. Hailon (2011) dégage, de façon précise, les modalités du "progrès » dans une coupe synchronique, en 2002, de plusieurs titres de la presse française, de formulations issues de l'extrême droite : l'extension, par exemple, de «forces de sécurité », en remplacement de «forces de l'ordre/de police » se manifestant sur le mode de la reprise réticente (dans Le Monde), c'est-à-dire comme « discours autre », ou bien comme formulation « naturalisée », énoncé sur le mode de l'évidence (dans Le Figaro).

Dès lors que l'on met en regard les deux plans où se joue l'altérité discursive dans un Discours, celui de l'autre représenté ( $\boldsymbol{E}^{\prime}$ ) se « détache », comme en relief,

$10 \mathrm{Au}$ delà de cette très sommaire évocation, je renvoie à la fine analyse d'ensemble de C. Rannoux des guillemets d'emprunt et au complexe travail d'identification et d'interprétation de leur valeur prise dans les contradictions - et contorsions - discursives de Léautaud rejetant un discours vichyssois qui « le parle». 
sur le fond de celui de l'autre, constitutivement présent $(\boldsymbol{E})$. Dans un Discours - constitutivement hétérogène - la RDA opère un partage entre ce que métalangagièrement elle institue, sélectivement, comme dire autre, et ce qui, complémentairement, s'y trouve donné - quels que soient les autres qui y travaillent, comme « propre ». Le mouvement de différenciation d'avec « de l'autre » que marque la RDA suppose celui, non-dit, d'une assimilation, incorporation, de l'autre - complémentaire - « fait sien ». C'est par ce double mouvement que passe la fonction dynamique de la RDA dans le discours : celle de la production métadiscursive d'une image configurant l'un - discursif, textuel, subjectif - par ses autres, désignés comme tels.

Une lecture réglée - d'analyse de discours, d'interprétation de textes... - ne saurait se passer de cette prise en compte des deux plans où, dans un discours, joue du discours autre : celui, saillant de l'autre $\boldsymbol{E}$ ', représenté comme tel, certes, mais profilé sur le fond de l'autre, $\boldsymbol{E}$, présent, constitutivement. Mais cette question du « traçage » de $\boldsymbol{E}$ ' dans $\boldsymbol{E}$ ne relève pas seulement d'une « visée » descriptive : c'est dans le mouvement spontané du dire qu'elle se pose, émergeant parfois de façon aiguë, pour les deux pôles de la co-énonciation, en tant que partie intégrante du sens, et de ses enjeux.

\section{Le prélèvement de $E$ 'dans $E$ au fil du dire : incertitudes et enjeux d'un tracé}

Chargée de sens et d'enjeux subjectifs, cette distinction que la RDA fait passer dans un Discours entre ce à quoi elle réfère comme « autre » et ce qui, par là, est institué différentiellement comme discours « de soi », n’opère pas un partage tranché : c'est à la fois dans son univocité et dans sa discrétude que le tracé selon lequel, dans un Discours, la représentation se démarque de la présence du discours autre, se trouve interrogé - discuté en malentendus, estompé en continuum ou questionné comme indécidable.

Les incertitudes de la « découpe » métalangagière opérée par la RDA dans la matière déjà-dite du dire - où se joue, on l'a dit, pour (et entre) les co-énonciateurs, une part du sens de ce dire - proviennent de deux traits négatifs propres au rapport entre discours autre représenté et présent :

- d'une part, le statut de représenté ( $\left.\boldsymbol{E}^{\prime}\right)$ n'est pas synonyme d'explicite ou de marqué, il y a donc du représenté implicite... (cf. ci-dessous $2.1,2.2,2.3$ )

- d'autre part, le statut de non-représenté $\boldsymbol{E} \backslash \boldsymbol{E}^{\prime}$ : complémentaire de $\boldsymbol{E}$ ' dans $\boldsymbol{E}$ (correspondant à ce qui se donne comme parole propre) n'est pas synonyme de « présence insue », il y a de l'autre consciemment énoncé comme « fait sien ») (cf. ci-dessous 3) 
C'est un champ complexe qui est ouvert par cette incertitude du « traçage » de l'autre représenté, mettant en jeu :

- la dualité dissymétrique des interlocuteurs - et l'irréductible différence de leur mémoire discursive,

- le travail interprétatif - et ses aléas,

- l'intentionnalité - et ses marges indécises,

- la conscience de l'énonciateur - et ses degrés.

Ce qui suit ne vise qu'à y placer quelques repères... ou questionnements.

\subsection{La RDA à marquage zéro}

Représentation n'implique pas « marquage » : ce sont, on l'a $\mathrm{vu}^{11}$, tous les modes de RDA qui présentent, à l'extrémité du « dégradé » de marquage des formes par lesquelles ils se réalisent, une réalisation à marquage zéro, relevant purement de l'interprétation en discours, comme dans les énoncés suivants ${ }^{12}$ :

\section{MAE}

(1) Probablement, puisque je ne cherchais pas des manuscrits, aurais-je pu trouver les mêmes livres ailleurs, ou les faire venir par la bibliothèque de mon université. Mais \{longtemps je m'étais couché de bonne heure\} en rêvant de monter à la Montagne Sainte Geneviève avec Abélard, de me rendre [...] [U. Eco, Discours de réception comme Docteur Honoris Causa de l'Université Paris 3, 20.1.1989 ${ }^{13}$.

(2) Dès qu'une femme franchit la frontière du territoire masculin, la nature du combat professionnel change. \{Les vertus qu'on exige alors d'une femme, on se demande combien d'hommes seraient capable de les montrer\} [F. Giroud, Si je mens..., 1972] ${ }^{14}$.

\section{MAS}

(3) Selon Naouri, le parent n'a pas à donner de justification et à entrer dans l'éventuel cycle de la négociation. \{L'enfant doit être « tenu », c'est la seule condition pour qu'il se structure.\} [F. de Singly, Libération, 27-04-2004].

$11 C f$. chap. 9.3.2 «le niveau de marquage ».

12 Voir aussi, par exemple : chap. 4 ex. (71-77) pour le Bivocal; ou chap. 9 ex. (19-26) pour les « extensions » de DI et MAS. et pour l'allusion (i.e. la MAE à marquage zéro) Authier-Revuz (2000a) et (1995/2012 : 272-296). Les segments donnés à reconnaître dans les exemples ci-dessous sont repérés par des \{\} .

13 "Longtemps je me suis couché de bonne heure », incipit de Du côté de chez Swann (Proust). 14 « Aux vertus qu'on exige dans un domestique, Votre Excellence connaît-elle beaucoup de maîtres qui fussent dignes d'être valets ? " [Beaumarchais Le Barbier de Séville : Acte I, sc. 2 (FIGARO)] 
DI

(4) Quatre jours après la mère revient et me dit que l'enfant est plus calme. \{Sans gardénal elle a dormi, d'un sommeil léger, mais sans cauchemars, l'énurésie a persisté les deux premières nuits et on n'a pas grondé l'enfant. Depuis deux jours, l'incontinence nocturne a cessé, l'appétit est revenu et l'enfant est gaie dans la journée. Elle pose de nombreuses questions.] [F. Dolto, Psychanalyse et pédiatrie, 1971].

DD

(5) Sur le soir du 11 août, par la grande chaleur humide et malsaine des étés de Versailles, Jeanne survient plus effervescente que jamais, \{tout va de mieux en mieux, je sors de chez la Reine qui est très contente, et maintenant laissez-vous faire...] [C. Manceron, Les hommes de la liberté, IV, p. 104, 1979].

\section{Bivocal-DIL}

(6) Valentin $n_{\mathrm{i}}$ nie éperdument et prétend être resté couché chez lui $\mathrm{i}_{\mathrm{i}}$ toute la nuit. \{Et d'abord : comment Mme Ridel aurait-elle pu le $\mathrm{e}_{\mathrm{i}}$ reconnaître ? la nuit était sans lune et la rue très mal éclairée.\} [A. Gide, Souvenirs de la Cour d'assises, Pléiade, p. 645].

En l'absence de forme (segmentale, typographique, intonative) marquant métalangagièrement comme discours autre - «pas de soi » - un élément du Discours, rien ne distingue, formellement, sur le fil du Discours, ce qui de l'ailleurs discursif y relève de la présence ou de la représentation, c'est donc à l'interprétation - sur la base d'indices discursifs - de faire la différence, essentielle quant au sens attribué au Discours, entre ces deux statuts :

- élément de discours qui, dans ce qui s'énonce comme Discours « de soi », est identifiable comme trace de présence de discours autre,

- élément de discours énoncé métalangagièrement comme discours autre et intentionnellement donné à reconnaître comme tel (par des indices) ${ }^{15}$.

Là où le premier relève du "risque » inhérent au langage, à se produire et à se recevoir constitutivement, dans l'extériorité discursive des " mémoires " des co-énonciateurs (à la fois dissemblables et échappant à leur contrôle), le second procède, pour l'énonciateur, d'un choix du risque, en « confiant » au récepteur le soin de reconnaître par lui-même la topographie, qu'il a implicitement dessinée dans son dire, de l'autre dans le propre.

15 C'est-à-dire correspondant à une forme de RDA à marquage zéro. E. Orlandi (2012: 89) souligne ainsi l'opposition entre ces deux statuts pour un discours autre non marqué comme tel : « L'allusion cesse d'en être une quand nous sommes parlés par l'idéologie, c'est-à-dire quand dans nos mots, d'autres mots parlent, qui produisent un effet dans notre dire au travers de l'oubli "idéologique" ». 


\subsection{Discours énoncé comme autre et donné à reconnaître par des indices}

Le travail interprétatif d'identification d'un élément $\mathrm{X}$ donné à reconnaître comme « de l'autre » repose sur des indices :

- intradiscursifs, impliquant l'environnement, immédiat ou non, du segment concerné ;

- interdiscursifs, impliquant les «mémoires » de $\mathbf{L}$ et de $\mathbf{R}^{16}$;

- contextuels, impliquant une connaissance de la situation et des sujets parlants, $\tan \mathbf{L}$ que les « $\boldsymbol{l} »$.

En fonction du degré de saillance de chaque indice et de leur cumul éventuel, les agencements discursifs observables donnent forme très diversement au choix du non-marquage, entre la quasi-annulation de l'aléa qu'il comporte ou au contraire sa mise en jeu maximale intensifiant, avec le risque d'échec, les bénéfices de la connivence.

De la diversité des fonctionnements propre à chaque mode de $\mathrm{RDA}^{17}$, se dégagent des mécanismes généraux de reconnaissance des formes non marquées reposant sur une mise en rapport, interprétative, de l'élément $\mathrm{X}$ en question avec un autre élément de discours. Schématiquement, dans un jeu à quatre termes, $\mathrm{X}$ sera reconnu comme « autre » à travers son

- rapport de (A) discordance avec le Discours en train de se faire de $\mathbf{L}, v s$ (B) concordance avec de l'ailleurs,

- établi (1) sur le fil de l'intradiscours ou (2) dans l'espace de l'interdiscours.

16 « Mémoire » est pris ici au sens particulier de mémoire interdiscursive ( $c f$. ci-dessus, chap. 10 1.4.1, p. 389 et 2.1.2.(i), p. 401, étant entendu que la mémoire, au sens courant, y est requise comme aussi dans la prise en compte de "l'avant" du fil, intradiscursif, ou des connaissances liées au contexte situationnel.

17 Le fonctionnement des indices permettant d'identifier un discours autre donné à reconnaître dépend du mode de RDA réalisé en marquage zéro : les conditions d'identification (ou non) d'une allusion (MAE non marquée) associée inopinément au déroulement du Discours ne sont pas comparables, par exemple, à celles d'un DD ou d'un Bivocal non marqué, attribuable à un $\boldsymbol{l}$, personnage d'une narration. Aussi la description précise des mécanismes par lesquels passent, spécifiquement pour chacun des modes, la reconnaissance de ses réalisations non marquées - ici, très majoritairement, illustrée dans le cas de la MAE non marquée, « allusive », $c f$. Authier-Revuz (2000a) - devrait s'inscrire dans le cadre de l'étude détaillée $\mathrm{du}$ fonctionnement de chacun d'eux, qui n'est pas (comme on l'a vu chap. 9) l'objet de cet ouvrage. 


\begin{tabular}{|l|l|l|}
\hline & A Discordance & B Concordance \\
\hline Intradiscours (1) & $\begin{array}{l}\text { A }_{1} \\
\text { ex. (1), (5), (7), (8) }\end{array}$ & $\begin{array}{l}\text { B }_{1} \\
\text { ex. (3) ; (4), (20), (21) }\end{array}$ \\
\hline Interdiscours (2) & $\begin{array}{l}\text { A }_{2} \\
\text { ex. (22) }\end{array}$ & $\begin{array}{l}\text { B }_{2} \\
\text { ex. (1), (2), (22) }\end{array}$ \\
\hline
\end{tabular}

\subsubsection{Indices dans l'intradiscours}

(i) Indices de discordance $A_{1}$

Parmi eux, on distinguera deux types :

a) d'une part, celui de diverses figures d'altérité, de différence, d'un élément $X$ relativement à son co-texte, différence attribuable interprétativement à l'appartenance à un discours autre, telles, par exemple :

- l'étrangeté de la survenue, dans l'exemple (1), d'une information concernant les heures de coucher d'un orateur évoquant un parcours universitaire dans un discours protocolaire (parfait exemple de ce que Riffaterre appelle la « trace de l'intertexte », cet indice qu'il y a « de l'autre » demande, pour aller au-delà de la simple perplexité, qu'un rapport de « concordance » avec l'incipit proustien puisse être établi) ${ }^{18}$;

- la rupture de « ton » - registre de langue et régime énonciatif dans l'exemple (5), entre le récit en $i l$, de style soutenu, du narrateur historien, et l'adresse familière en $j e / t u$, identifiée par là comme parole de $\boldsymbol{l}=$ Jeanne

- la contradiction interne à l'énoncé proposé par M. Pêcheux (1975 : 88) :

(7) Celui qui sauva le monde en mourant sur la croix n’a jamais existé.

comme exemple d'une hétérogénéité dont la reconnaissance conditionne l'acceptabilité du dire, ou, plus trivialement :

(8) Au fait, ça ne me gêne pas, je me promènerai tout aussi bien, mais tes chevaux, ce sont des mulets (oral, 10-09-2005).

b) d'autre part, celui de marques - en langue - de discordance, de différence ou d'un quelconque suspens méta-énonciatif qui, portant sur un élément X, sont susceptibles d'être interprétées comme celle de l'appartenance de cet $\mathrm{X}$ à un discours autre.

18 Le contexte institutionnel du genre du discours doublant le co-texte au sens le plus strict. Pour d'autres exemples, voir Authier-Revuz (2000a) et (1995/2012, chap. 6.2.4). 
Tel est le cas du guillemet de modalité autonymique qui, on l'a vu (chap. 8.3.2), marquant qu'un élément du dire « ne va pas de soi » (MA), n'est pas pour autant une marque que cet élément « vient d'un autre discours » (MAE) : la non-coïncidence énonciative dont il est le signe peut, en discours - via l'intra ou l'interdiscours - se charger interprétativement d'un sens d'emprunt à un autre discours - fonctionnant alors comme un indice de MAE.

C'est ce qui apparaît, par exemple ci-dessous, dans l'énoncé (20) où c'est couplé à un fait de concordance dans l'avant du texte que l'élément " faire des mariages », guillemeté, est interprété comme emprunté à une instance autre que celle du narrateur ; ou dans la discussion suscitée par l'interprétation en (27) ci-dessous d'un guillemet de Proust, selon que la marque - différenciatrice - dont il affecte « étoiles nouvelles » est, ou non, «transformée » (comme on le dit d'un essai au rugby!), c'est-à-dire comprise comme indice d'emprunt, par concordance avec l'ailleurs d'un poème de Heredia...

Deux formes, également méta-énonciatives, présentent un fonctionnement semblable à celui du guillemet de MA - une valeur en langue, étrangère à la RDA, mais apte à recevoir, en discours, le sens d'un renvoi à du déjà-dit : le point de suspension (et son correspondant prosodique) et " etc. ${ }^{19}$. Ces marques d'inachèvement délibéré du dire se prêtent, bien entendu, à l'appel à une mémoire discursive ${ }^{20}$ du destinataire à même de partager implicitement la " suite » tellement déjà dite qu'il n'est pas nécessaire de l'énoncer matériellement une fois de plus. L'énonciation - stéréotypique - des proverbes, paroles célèbres, rengaines diverses, notamment, emprunte volontiers ce mode connivent, " économisant » une redite intégrale superflue :

19 Une autre forme typographique - celle du trait d'union méta-énonciatif (et non lexical) affichant la « soudure » d'éléments normalement détachés, comme dans « Montrer, comme c'est le cas dans ce premier épisode, Berlioz et ses états d'âme (Dieu, que Mesguich est insipide et théâtral) en futur-génie-qui-lutte-contre-papa-et-maman ne représente qu'un intérêt minime [Télérama, nov. 1983, n¹764, p. 107] ", semble, elle, impliquer le renvoi au déjà-dit (figé) des éléments soudés (voir d'autres exemples in Authier-Revuz (1992 : 488). (Le trait d'union, comme image écrite d'un fait intonatif, peut, comme par exemple chap. 8 (3), être étranger au signalement d'un stéréotype).

20 Interdiscursive, comme dans les exemples notés ci-dessous, mais aussi bien intradiscursive, faisant jouer, comme le guillemet de (20), la complicité créée par un univers textuel commun ( $c$. une belle mise en œuvre dans l'évocation d'une atmosphère familiale peu à peu partagée avec le lecteur dans L'Amour des commencements de J.-B. Pontalis (1986 : 69, 114) évoqué in Authier-Revuz (1995/2012 : 287). 
(9) Difficile de discuter de son salaire, voire impossible. [...] Encore un des méfaits de la culpabilisation des femmes qui travaillent. Il est temps de prendre conscience que si l'argent ne fait pas le bonheur, etc., etc. [M.F. Sablé, Le nouveau F, juil.-août 1983, p. 13].

(10) Lorsque François Miterrand a soulevé, en mai dernier au Collège de France, la question d'une chaîne culturelle et éducative, il ne pensait sincèrement pas provoquer des réactions audio-visuelles aussi enthousiastes. Quand j'entends le mot culture, je sors... Eh bien pas du tout [L'événement du jeudi, 18.07.85, p. 77].

(11) [...] une partie du sort de la planète est désormais suspendue aux rebondissements de ce vaudeville qu'on ne doit pas sous-estimer : le nez de Cléopâtre et le zizi de Clinton, s'ils eussent été plus courts, etc. D’ailleurs, déjà, les palestiniens s’inquiètent. [Libération, 23.1.1997, affaire Lewinski-Clinton]

Si courants qu'ils soient, les fonctionnements de ces formes associées à du déjàdit à reconnaître, sont ceux d'indices de RDA et non de « marques », comme le montrent par différence :

- d'une part, les emplois de ces formes ne comportant pas la mise en jeu d'un énoncé déjà dit, mais seulement, par exemple, celle d'un savoir encyclopédique pour etc. (12), ou l'invite faite au destinataire de « rejoindre » interprétativement l'énonciateur dans l'espace de non-dit qu'il ouvre (13)

(12) C'était un fouillis indescriptible, livres, vêtements, chaussures, balles de tennis, disques, photos, etc. Il a renâclé mais il a fini par s’y mettre [oral, 20.09.1992].

(13) J'ai reçu ce matin une lettre de Bertrand... Je voulais vous la montrer, il est follement heureux chez vous... Il me parle de votre mère... Cela ne m'étonne pas qu'elle soit bonne et charmante... [Mauriac, Asmodée III-10, cité par Grevisse Le bon usage].

- d'autre part, à l'inverse, des formes suspensives renvoyant univoquement à un discours autre, telles :

(14) Barcelone avait posé sa candidature en 78, trois ans avant Paris. Rien se sert de courir, vous connaissez la suite [ $\underline{\mathrm{FR} 3}, 17-10-86$ (Echec de la candidature de Paris aux jeux olympiques de 1992)].

(15) Il paraît que c'est la base de leur fortune. Bien mal acquis, tu connais la chanson, eh bien, c'est pas si clair que ça ! [conversation, 3.10.2010].

Au-delà, lieu sensible de l'extériorité interne au discours, où s'articulent le constitutif et le représenté, c'est tout un ensemble, non délimité, des formes retenues - au-delà du « discours rapporté » identifié à la trilogie DD-DI-DIL - en tant qu'elles « portent de l'autre » dans le dire de l'un, qui a été, à travers de très nombreuses études, un objet privilégié de l'AD, comme des perspectives dialogique ou polyphonique $^{21}$. Dans cet ensemble nous retiennent ici les formes susceptibles de jouer le

21 Il n'est pas question de rendre compte, ici, du détail des analyses portant sur les formes particulières - faits de pré-construit/présupposition, subordination, focalisation ou clivage, néga- 
rôle d'indice dans la reconnaissance, interprétative, d'un élément comme relevant d'un discours autre - c'est-à-dire dont la valeur, en langue, est compatible, voire propice, au plan du sens en discours, avec la mise en jeu d'une instance de discours différente de l'énonciateur $\mathbf{L}$, à l'exclusion de celles qui, la contraignant, en apparaissent comme des marques, partie prenante, de ce fait, du champ de la RDA ${ }^{22}$.

Ainsi, par exemple, de la tournure ce n'est pas $X$ mais $Y$ : elle est analysée par Courtine (1981a) comme site d'inscription, en X, d'un déjà-dit dans un énoncé dit par là " divisé », c'est-à-dire qui est - relativement à une mémoire discursive - interprété comme "divisé » entre le dire de l'un et un dire autre - dire autre dénonçant une tactique opportuniste du parti communiste, dans l'énoncé communiste cité par Courtine :

(16) Notre politique à l'égard des chrétiens n'a rien d'une tactique de circonstance, c'est une politique de principe.

De même la lecture, résolument recontextualisante, que P. Sériot (2007) fait de Bakhtine, dans l'environnement discursif qui était le sien, lui permet-il de dégager, interprétativement, dans la récurrence observable du schéma « X n'est pas $Y$ mais $Z$ » l'insistante mise en cause polémique, en $Y$, d'un discours autre non explicité comme tel :

[...] non seulement Bakhtine ne donne pas la parole à l'autre pour qu'il puisse se défendre, mais encore il ne nomme pas son adversaire principal, privé du droit à la parole et à l'existence par le nom. En effet, lorsque Bakhtine dit « X n'est pas Y mais Z » Y est la plupart du temps une crypto-citation de son adversaire de tout temps, V. Vinogradov (1894-1969).

Mais si la valeur en langue de cette forme d'opposition entre deux éléments prend aisément corps en discours, dans l'opposition à un autre discours, ce chemin interprétatif n’est pas contraint : un énoncé tel que

tion, connecteurs... - objets largement partagés par les diverses approches, ni des différences de traitement entre celles-ci ou dans leurs variations internes. Je reviens, de façon fragmentaire et schématique ( $c f$. ci-dessous Remarque 1) sur quelques points.

$22 C f$., par exemple, dans ces formulations, la caractérisation nette d'éléments comme « ouvrant » sur la mise en jeu d'un discours autre, mais non le « marquant » : S. Moirand (2005), évoquant « certaines constructions qui semblent favoriser, sémantiquement ou syntaxiquement, l'inscription des dires antérieurs ou des dires d'autrui (voir, par exemple, Bres 1998, 1999) ce qui nous renvoie à la notion de pré-construit de l'analyse du discours française (Henry 1975 par exemple) »; ou Combettes (2014), relevant, en diachronie, parmi les formes de dialogisme accompagnant l'émergence du texte argumentatif « le statut périphérique des constructions topicalisées [qui] peut être considéré comme un indice du dialogisme sous-jacent ». 
(17) H. n'est quasiment d'aucune aide, ce n'est pas qu'il soit de mauvaise volonté mais il ne « comprend pas » ce qu'il y a à faire ! [Corr. privée, dans le cadre d'un déménagement, 26-08-2013].

« divise », certes, la pensée et le dire du scripteur entre les divers points de vue envisageables à ses yeux, mais c'est sans qu'il y ait ici à faire intervenir une quelconque trace de « discours autre » (sinon, bien sûr, l'extériorité discursive habitant, constitutivement, tous les mots de tous les énoncés... $)^{23}$.

De même, si en (18) puisque, compte tenu de son contexte, introduit bien la reprise - polémique - d'un dire de Jean se vantant d'avoir suivi un discours en allemand :

(18) Puisque Jean a tout compris ce que ce type a dit, il va pouvoir nous expliquer...

en (19) en revanche, c'est sur un constat fait par lui, hic et nunc, et non sur un dire autre que l'énonciateur fonde sa conclusion (de dîner sans attendre un convive hypothétique) :

(19) Puisque G. n’a pas téléphoné, on va commencer sans lui.

Les diverses formes reconnues comme indices - et non pas « marques " - de possible mise en jeu de discours autre peuvent, régulièrement, figurer dans des énoncés des types (16) à (19) : c'est en fonction de divers éléments co- et contextuels que leur valeur en langue - celle d'une structure de différenciation, opposition, décalage, discordance, altérité.... ${ }^{24}$ de « non-même » ou de « non-un » - se convertira, ou non, en discours, en mise en jeu d'une altérité spécifique, celle d'un ailleurs discursif. Ainsi, ces formes ne sont-elles pas des formes linguistiques de dialogisme - comme le sont les formes de la RDA. Mais il relève bien de la langue et de sa foncière abstraction d'offrir une diversité de « structures d'altérité » - négative, comparative, concessive, etc. - impliquant « de l'un et de

23 Ceci constitue un point de clivage théorique net entre, d'un côté, $\mathrm{AD}$ et dialogisme au sens où il a été pris ici, ainsi que chez J. Bres ou S. Moirand, par exemple, et de l'autre, polyphonie intralinguistique (Ducrot et Scapoline) où les « voix » des " personnages de l'énonciation », purs « êtres discursifs ", n’ont pas à être présupposées comme actualisées ( $c f$. sur ce point les réflexions de Rabatel 1990 : 6, de Perrin 2005 et de Sitri 2015). Ainsi Bres et al. (2006 : 31) soulignent-t-ils le fait que « le dialogisme d'un énoncé (E) tient à ce qu'il "répond" à un énoncé [e] qu’il présuppose actualisé en le "reprenant" de diverses façons, allant de sa citation à sa présupposition. ».

24 Cf. encore, dans Ducard (2004) (cité in Sitri 2015a), la concession, traitée en termes culioliens, de « forme schématique » comme faisant fonction de « marqueur de discontinuité ». 
l'autre » (l'autre ne se ramenant pas à de « l'autre dire »), comme autant de moules ou de patrons permettant de donner forme au rapport d'un discours à ses autres.

On distingue ainsi entre ces deux modes de mise en jeu d'un discours autre dans un énoncé : assuré - c'est-à-dire marqué - dès le plan de la langue, ou bien résultant de la saturation, en discours, par du « discours autre », de la relation - abstraite - d'altérité ${ }^{25}$, telle que, diversement, elle est configurée au plan syntactico-sémantique dans une variété de formes de langue, qui apparaissent dès lors comme autant de « sites d'accueil » pour un possible « discours autre ». Cette distinction est en accord étroit avec le cheminement de F. Sitri dans ses analyses $\mathrm{du}$ fonctionnement concessif ${ }^{26}$ : menée dans un cadre non polyphonique, relevant d'une $\mathrm{AD}$ ouverte sur la théorisation culiolienne ${ }^{27}$, sa réflexion, qui rejoint celle de S. Mellet (2009), aboutit à préciser, à propos de certes, la distinction formulée ici en termes de marque vs indice :

Nous ne parlons donc pas de « marqueur dialogique, c'est-à-dire de « morphème dont le signifié en langue programme la signification dialogique (Bres et Mellet 2009: 6 note 4) mais de marqueur susceptible en discours de produire des effets dialogiques ou, pour reprendre l'expression de $\mathrm{S}$. Mellet dans son article, de « morphème(s) à fort potentiel dialogique» [...]. (Sitri 2015a : 63)

Remarque 1 : Marqueurs et indices dialogiques. L'appellation « marqueurs dialogiques » sous laquelle J. Bres réunit, dans le souci de saisir le " phénomène du dialogisme dans son unité ${ }^{28}$ une série de formes « extrêmement fournie et non close » où le renvoi à du discours autre passe par des " signifiants hétérogènes » et un " mode d'être très variable (de l'explicite clairement marqué à l'implicite laissé à l'interprétation ${ }^{29}$ n'est évidemment pas à entendre au sens - opposé à « indice » - donné ici à « marque ». De fait, ces « marqueurs dialogiques » incluent - au-delà des formes univoques de renvoi à un discours autre - non seulement des éléments de langue, susceptibles ou non de recevoir, en discours, une interprétation dialogique, mais n'im-

25 Je renvoie ici à la réflexion importante de Culioli, à propos de l'opération de négation, posant « l'altérité » comme « de fondation » (Culioli 1988/1990 : 97) et associant la négation à la construction par le sujet d'une « relation d'altérité », et à « la représentation des possibles ». (ibid. : 113).

26 Garnier et Sitri (2009), Sitri (2015a).

27 Cf. notamment De Vogüé (1992), Ducard (2004), Paillard (2011).

28 Perspective particulièrement explicite dans Bres et Vérine (2003), Bres (2005b). On notera que contester le terme de "marqueur dialogique ", en ce qu'il rassemble les formes signifiant univoquement de l'autre discours et celles qui sont seulement aptes à recevoir cette interprétation, ne met nullement en cause l'intérêt de la position de J. Bres, soulignant de façon très bakhtinienne l'unité du fait dialogique; cela ne fait qu'insister sur un paramètre - à mon sens essentiel - de cette diversité « des façons dont se signifie le dialogisme de l'énoncé » que Bres appelle à décrire.

29 Bres et Nowakowska (2006) et article « Dialogisme - marqueurs de » in Détrie et al. (2001). 
porte quel segment de discours, dès lors que, dans une mémoire discursive donnée, il serait reconnu comme écho d'un ailleurs ( $c f$. ci-dessous 2.2.2), ce qui annule d'emblée toute entreprise de « listage » si ouvert soit-il.

Ainsi, le frappant exemple d'allusion - interdiscursive - à l'Éducation Sentimentale par J. Echenoz proposé comme « marqueur de dialogisme » (p. 88 de l'article du même nom in Détrie et al. (2011)) de type « détournement » ne présente, si insistant soit-il «pour qui a lu Flaubert », aucun élément qui, en lui-même, ferait signe vers de l'ailleurs... Ni marque univoque, ni indice interne de renvoi à de l'ailleurs discursif dans cet énoncé :

Il connaît la mélancolie des restauroutes, les réveils acides des chambres d'hôtel pas encore chauffées, l'étourdissement des zones rurales et des chantiers, l'amertume des sympathies impossibles [J. Echenoz, Je m'en vais, Éd. de Minuit, 1999, p. 196].

pas plus que dans ces autres allusions (parmi bien d'autres...) au même texte :

Il connut les longues attentes des aéroports, les réveils poisseux sous le ventilateur, l'étourdissement des déserts et des mégapoles, l'amertume des connexions interrompues [R. Debray, Le bel âge, éd. Café Voltaire, 2013].

Il connut la mélancolie des premiers matins, les froids réveils aux portes des usines, l'étourdissement des cités ouvrières et des bidonvilles, l'amertume des sympathies interrompues [M. Schneider, $2010:$ 17).

C'est seulement de leur rencontre (aléatoire, même si elle est évidemment visée par l'auteur) avec l'ailleurs flaubertien qu'ils pourront être reconnus dans le jeu de leurs consonances (rythmiques, syntaxiques, lexicales) et de leurs dissonances comme écho sarcastique de la mélodie désabusée du parcours de Frédéric :

Il connut la mélancolie des paquebots, les froids réveils sous la tente, l'étourdissement des paysages et des ruines, l'amertume des sympathies interrompues [G. Flaubert, l'Éducation sentimentale, partie 3, chap. 6].

En très large proportion, les formes analysées - de négation, interrogation, concession, opposition, comparaison, confirmation, tours corrélatifs, nominalisation, relatives... - apparaissent non comme des formes renvoyant par elles-mêmes à un discours autre (telles il dit, selon, paraît-il, officiellement, officieusement...) mais comme des formes susceptibles, en contexte, de se charger de cette dimension d'altérité discursive. C'est d'ailleurs de façon répétée que J. Bres pointe la dimension de potentialité dialogique des « marqueurs » dans des commentaires tels que :

les vertus dialogiques de cet outil [négation] [...]. [...] la subordination [...] s'avère particulièrement apte à porter le dialogisme [...]. [...] La subordination avec thématisation de l'élément subordonné fait partie de ces tours qui permettent à E1 de prendre en compte la parole de e1, même si c'est pour s'opposer à elle (Bres 1999 : 73, 81, 83).

[...] complétives, circonstancielles, relatives peuvent être des outils de dialogisme (Bres 2005).

et à la question - cruciale quant au statut de ces formes, comme forme dialogique en langue, ou forme se prêtant à un sens dialogique en discours - posée à propos de mais : 
Dans quelle mesure mais pose-t-il lui-même l’altérité énonciative?

c'est par la négative qu'il répond :

Si dans certaines occurrences [de X mais $\mathrm{Y}$ ] X n'est pas nécessairement dialogique, dans d'autres il l'est parfaitement. (Bres 1999 : 80)

Et, très normalement, les énoncés proposés comme illustration - convaincante - de fonctionnement dialogique de ces formes comportent, largement, des éléments co-textuels ou sont assortis d'informations contextuelles renforçant ou affirmant la mise en jeu d'un discours autre : tels, cumulés à une variété de tours négatifs (Bres 1999 : 74-75) dont ils assurent l'interprétation dialogique, guillemets, conditionnel, lien intra ou interphrastique avec une forme explicite de RDA, situation polémique, par exemple.

A l'intérieur du champ dialogique tel que Bres le parcourt, à travers la variété de ses « marqueurs, « de la citation à la présupposition », la distinction entre formes imposant (I) vs permettant (II) la mise en jeu d'un discours autre apparaît suffisamment sensible pour que le tracé de la frontière entre les deux soit - au delà du cas privilégié de la négation - l'objet d'un questionnement récurrent dans le cadre dialogique comme dans le cadre polyphonique ${ }^{30}$.

Ainsi, si on considère le secteur des formes dédiées à la « confirmation », dont Bres (1999 : 78) propose, par exemple, une liste ouverte

\section{bien, évidemment, décidément, effectivement, sûrement...}

le souci de spécifier le caractère « polyphonique en soi » ou non, de tel ou tel élément apparaît-il régulièrement, affinant - et déplaçant - au fil des études la frontière entre les ensembles (I) et (II) : Rubattel (1990), par exemple, argumente pour ranger évidemment du côté (II) ; cependant que C. Rossari (2007) étudiant le couple effectivement/en effet dégage clairement, par la mise en parallèle des deux " profils », ce qu'ils partagent - le fait que chacun

confirme le bien fondé de l'attitude épistémique $[\Delta]$ adoptée à propos d'un état de chose préalable à l'énonciation de la forme adverbiale (Rossari 2007 : 127)

et ce qui les distingue - la propriété supplémentaire requise pour effectivement (I) que formule, au point $[\Delta]$ ci-dessus, l'ajout selon lequel l'attitude épistémique confirmée est nécessairement celle

qu'une instance - distincte du locuteur au moment de l'énonciation de l'adverbe - a adoptée (ibid.)

De même, la ligne de partage proposée, dans le cadre polyphonique, par Perrin (2005) entre les « marqueurs » seulement « potentiellement polyphoniques » (II) (comme : morphème de néga-

30 Cf. par exemple Kerbrat-Orecchioni (1980 : 164 sq.), Rubattel (1990) ou Perrin (2005), contestant le caractère « polyphonique en soi » d'éléments tels que : négation, si hypothétique, évidemment, connecteurs argumentatifs... 
tion, si hypothétique, évidemment), et les « marques polyphoniques [...] fortes ou directives qui imposent en soi une séquence écho » (I), parmi lesquelles, aux côtés de il paraît (que), selon $l$, comme dit $l$, il range certes, se trouve-t-elle à nouveau déplacée par les études évoquées ci-dessus qui, de façon convaincante, contestent le caractère (I) de «marqueur dialogique » de certes, pour le redéfinir comme (II) marqueur d'altérité, susceptible de fonctionnement dialogique.

\section{(ii) Indices de concordance $B$}

Les indices de rapport de concordance établis dans (1) l'intradiscours peuvent, notamment, relever :

- de la cohérence séquentielle immédiate entre une forme de RDA marquée et l'élément qui la suit, interprété comme RDA non marquée, relevant du même mode, comme dans les « extensions » de MAS ou DI en (3), (4), par exemple, ou d'un mode différent, selon les très classiques agencements d'un DI marqué suivi d'un DD ou d'un Bivocal non marqués ;

- de la ressemblance ou de l'identité entre un élément et une forme de RDA marquée figurant à distance dans le co-texte antérieur, et sollicitant la mémoire - intradiscursive - du déroulement du Discours. C'est le cas, dans les récits, des DD non marqués dont la reconnaissance - en tant que DD, et d'un certain $\boldsymbol{l}$ - s'appuie sur la similitude (de contenu et de manières de dire) avec des passages antérieurs marqués comme DD d'un $\boldsymbol{l}$ déterminé ; ça l'est aussi pour les MAE non marquées ${ }^{31}$, reprises allusives reposant sur une connivence intratextuelle avec le lecteur, se prêtant à de multiples mises en œuvre textuelles : que ce soit dans un bref article, avec, par exemple, l'effet rhétorique de «boucle » d'une conclusion reprenant, sur un mode non marqué, la RDA marquée en introduction; dans un texte argumentatif comme stratégie polémique de reprise « en contexte hostile » des mots préalablement cités de l'adversaire ${ }^{32}$; ou, dans le temps d'un long récit et de sa mémoire - celle des personnages et du narrateur offertes en partage au lecteur... comme en

31 Le travail de reconnaissance par écho intradiscursif jouant tant pour les éléments ne présentant aucune marque que pour des éléments marqués comme MA (par des guillemets) qui sont à reconnaître comme MAE. Je renvoie pour des exemples d'échos à courte ou à longue distance à Authier-Revuz (1995/2012 : 284-287) ou (2000a).

$32 \mathrm{Cf}$. par exemple l'importance des échos non marqués, donnés à reconnaître comme relevant du comme vous dites sur la base des diverses formes marquées de RDA - Vous dites " ... », selon vous « ... » - qui les précèdent, dans le discours offensif de Luce Irigaray adressé à « Messieurs les psychanalystes » sous le titre « Misère de la psychanalyse » (étudié in Authier-Revuz 1995/2012: 225-231). 
use largement Proust, par exemple, au fil des temps perdus et retrouvés ${ }^{33}$. Ainsi, dans « Un amour de Swann » :

(20) Hélas! il y aurait aussi le peintre, le peintre qui aimait à « faire des mariages », qui inviterait Forcheville à venir avec Odette à son atelier (p. 286).

faisant écho dans la mémoire irritée de Swann - et celle du lecteur - quelques temps et quelques dizaines de pages après, à la confidence faite par le peintre « dans l'oreille du docteur Cottard» :

« Rien ne m’amuse comme de faire des mariages » (p. 202).

ou cette formulation de la pensée de Mme Verdurin par le narrateur :

(21) [...] Mme Verdurin à qui le Président de la République apparaissait comme un ennuyeux particulièrement redoutable parce qu'il disposait de moyens de séduction et de contrainte qui, employés à l'égard des fidèles, eussent été capables de les faire lâcher (p. 217).

qui ne trouve sa saveur de condensé caricatural que précédée des nombreuses occurrences guillemetées de « ennuyeux », « fidèles », « lâcher » qui ont marqué l'initiation de Swann et du lecteur au « parler Verdurin ».

\subsubsection{Indices de rapport avec l'interdiscours}

Contrairement au repérage des indices intradiscursifs ${ }^{34}$, le fonctionnement des indices mettant en jeu - discordance ou concordance - le rapport entre l'énoncé et une extériorité interdiscursive repose sur une rencontre des mémoires interdiscursives de $\mathbf{L}$ et de $\mathbf{R}$.

\section{(i) Rapport de discordance}

Ainsi en va-t-il pour le rapport (A) de discordance qui peut aussi s'établir (2) (voir p. 430) entre un énoncé et sa mémoire interdiscursive, sous les espèces, par

33 On trouve dans Funakoshi-Teramoto (2005) et Moricheau-Airaud (2008b) des études précises, respectivement chez Flaubert et chez Proust, des échos intratextuels circulant dans l'écriture romanesque.

34 L'interprétation des « indices » de discordance intradiscursifs par exemple peut évidemment passer par la mémoire interdiscursive ( $c f$. le guillemet de MA, par exemple, pour l'interpréter comme (1) emprunt, (2) à tel discours autre), mais l'indice - comme une « question » - est d'emblée repérable sur le fil du discours lui-même. 
exemple, d'une incompatibilité entre un élément $\mathrm{X}$ du dire et la connaissance que possède $\mathbf{R}$ au sujet de $\mathbf{L}$ (et de son discours antérieur), comme dans

(22) Ben, on peut quand même pas faire stagner les bénéfices des actionnaires! [commentaire intonation neutre, en famille, d'une annonce de délocalisation d'usine, au journal télévisé 09-2010].

Par rapport à la caractérisation générale de l'ironie comme phénomène « foncièrement contextuel » en ce qu'il suppose une « discordance par rapport à la parole attendue dans tel type de situation $»^{35}$ ou comme reposant sur la formulation par L d'une « position dont on sait par ailleurs [...] qu'il la tient pour absurde » (Ducrot 1984 : 221), ce qui spécifie le type d'ironie jouant en (22), c'est que c'est exclusivement sur fond d'interdiscours - sans indices internes à l'énoncé, ni rapport contre-factuel à la situation comme pour «Quel beau temps ! » énoncé sous une pluie battante ${ }^{36}$ - que peut apparaître la distance métalangagière ${ }^{37}$ ironique d'un « comme dirait ou disait un autre » : par reconnaissance combinée de la discordance d'avec les extérieurs du déjà-dit de $\mathbf{L}$ et d'une concordance avec ceux du discours du libéralisme économique. Plus encore que la simple allusion ( $c f$. (24)) à un discours autre dont la « perte » appauvrit - parfois gravement - le discours, ce type d'ironie est, de la part de $\mathbf{L}$, le choix d'un risque radical de « contre »-sens dans l'interlocution, dans un jeu avec l'image que $\mathbf{R}$ se fait de $\mathbf{L}$, source du plaisir d'une complicité réassurée, de l'embarras d'un échec, ou du malaise créé, par une pratique excluante ou sécurisante - perverse ? - de l'ironie dans un groupe de personnes se connaissant à peine, c'est-à-dire privé des moyens de la reconnaître à coup sûr ${ }^{38}$.

35 Article «Ironie » in (DAD 2002 : 330).

36 Ce qui n'interdit pas de réunir les deux cas, en considérant que « la parole attendue » dans une certaine situation (et son « envers », la parole ironiquement tenue à distance) ne l'est que relativement à une «normalité » - interdiscursive - de ce qu' « on dit », ce qui « se dit » dans la dite situation.

37 Cf. le traitement de l'ironie comme «mention » par Sperber et Wilson (1978), et sa reformulation en modalisation autonymique par Basire (1985).

38 Tel, au cours d'un dîner, le flottement de convives non familiers les uns des autres, ne sachant comment « enchaîner » sur l'affirmation, proférée sur un ton « neutre », en réponse à une remarque évoquant la pratique des électrochocs comme heureusement dépassée : « non, non, les électrochocs, ce n'est pas si mal, d'ailleurs on y revient » [25-01-2014 ]. 


\section{(ii) Indices de concordance}

Les indices (B) de concordance s'établissent, sous l'espèce d'une nécessaire zone de mémoire partagée par $\mathbf{L}$ et $\mathbf{R}$ dont l'élément $X$ non marqué, donné à reconnaître comme un écho par l'un, est reconnu comme tel (c'est-à-dire comme écho intentionnel) par l'autre. C'est la rencontre des deux mémoires - énonçante et interprétante - en un point X de la chaîne qui l'établit comme RDA à marquage zéro : apportant à la " question » ouverte par un indice de discordance la " réponse » d'une source autre identifiée - incipit de La Recherche pour (1), phraséologie chrétienne pour (7), discours usuel de « l'économie de profit » pour (22) -, ou bien assurant à elle seule le statut de discours autre donné à reconnaître à un élément non repérable comme « discordant » sur la chaîne comme en (2) ${ }^{39}$.

Fonctionnant «à la connivence » d'un partage de mémoire, le mécanisme de l'allusion ${ }^{40}$ est à l'œuvre (couplé ou non à de la discordance) dans la plus grande variété de discours : échanges privés, médias, essais politiques, théoriques, critique littéraire... ; ressort des mises en résonances intertextuelles qu'orchestrent, densément, certaines écritures littéraires, telles, par exemple, celles de Proust ou de Perec ${ }^{41}$ - sans en exclure l'écriture poétique contrairement à l'a priori bakhtinien $^{42}$ - le dédoublement du dire par renvoi implicite à un ailleurs, atteint au procédé de fabrication dans le domaine des slogans, publicitaires en particulier et des titres de certains journaux ${ }^{43}$.

39 L'énoncé (2) rappelant que la « concordance » indice d'extériorité peut - au-delà d'une identité stricte - passer par la similitude formelle (syntaxique, sémantique, rythmique) d'un «patron » : $c f$. chap. 7, note 12, p. 231, ou ci-dessus, Remarque 1, les reprises « avec variations » de Flaubert par Echenoz et Debray.

40 Authier-Revuz (2000a).

41 Cf. Compagnon $(2000,2009)$ pour le réseau d'allusions - retrouvées ou perdues... - doublant le fil de La Recherche (voir ci-dessous (27) les « étoiles nouvelles »); ou Perec, chez qui la mise en texte d'emprunts non marqués - les « impli-citations », dit B. Magné (1989) - tient dans La Vie Mode d'emploi de « l'art du puzzle».

42 Cf. chap. 10.1.3, p. 385, comme le fait apparaître, par exemple, la partie consacrée à « L'intertextuel » (p. 49-89) dans Thomas (1989, La langue, la Poésie) et les strates intertextuelles, richement « dépliées » par J.-M. Adam, sous la surface d'un poème de Desnos, de deux vers d'Eluard, in (1999: 128 sq.), (2002 : 84 sq.), ou de la dernière phrase de Nadja d'A. Breton, in (2006 : 19 sq.). $43 C f$. par exemple Grunig (1980 : 115-145), Maingueneau (2000: $150 s q$.), ou le florilège qu'en propose la livraison de Tranel (Bonhomme et al. 2006) consacrée à « Interdiscours et intertextualité dans les médias », notamment chez Adam, Lugrin, Moirand, Revaz. 


\section{3 "Turbulences" aux frontières de la RDA dans le dire}

Il est possible d'évaluer, en soi, le degré de saillance d'un agencement d'indices - discordance/concordance - intradiscursifs présenté par un discours : on peut ainsi considérer que le « risque » du marquage zéro n'est pas loin d'être annulé par le jeu ostensible des indices, dans le discours, du partage des voix de l'un et de l'autre, tel par exemple, dans ce bivocal ${ }^{44}$ typique de Zola :

(23) Alors, elle lâcha tout ce qui lui vint à la bouche. Oui, oui elle n'était pas bête, elle voyait clair. On s'était fichu d'elle pendant le dîner, on avait dit des horreurs pour montrer qu'on la méprisait. Un tas de salopes qui ne lui allaient pas à la cheville [...]. Et la rage l'étranglant, sa voix se brisa dans des sanglots [Zola, Nana].

et que c'est en revanche « sans filet » - en l'absence d'indices intradiscursifs - que le contrepoint, en (2), qui, dans un dire dénonçant le pouvoir masculin, y inscrit la voix « pré-révolutionnaire » de Figaro est donné à reconnaître « confié » à la seule mémoire du récepteur.

Il en va évidemment tout autrement pour les indices interdiscursifs puisque leur degré de saillance est strictement fonction de la mémoire dans laquelle ils sont reçus : résonnant sur le mode de l'évidence dans une certaine mémoire, les échos de Proust, de Beaumarchais ou du discours sur les moteurs financiers des choix politico-financiers (en (1), (2), (22), par exemple), resteront « lettre morte » dans une autre. S'attachant à "L'intertextualité comme "mémoire de la littérature”, c'est ce que souligne T. Samoyaud (2010 : 68) en titrant une partie « Des indices variables pour un repérage instable ».

Ainsi, reposant sur des indices dont la saillance, variable pour ceux qui jouent sur le fil du discours, est aléatoire pour ceux qui engagent les mémoires des deux co-énonciateurs, la reconnaissance des formes non marquées introduit, dans la frontière entre soi et l'autre - telle que la « fixent » les marques de RDA de l'incertitude et du risque.

Diverses sont les formes que prennent les « incidents de frontière » qui apparaissent sur un mode : (1) de ratages et de conflits entre $\mathbf{L}$ et $\mathbf{R}$; (2) de doutes et d'incertitudes pour $\mathbf{R}$; (3) de flottements et « d'accidents » pour L lui-même.

44 Dans lequel jouent à plein notamment $\left(\mathrm{A}_{1}\right)$ discordance séquentielle immédiate (régime énonciatif, registre de langue...) entre narration et parole du personnage, $\left(B_{1}\right)$ cohérence immédiate avec la représentation d'un fait de parole (« lâcha tout ce qui lui vint à la bouche ») et concordance à distance, au plan du registre, avec d'autres propos explicitement représentés comme ceux de Nana. 


\subsubsection{Ratages et conflits entre $L$ et $R$}

Deux filières pour l'éclosion des ratages et conflits selon que le risque qu'ouvre le fait du non marquage apparaît comme celui que le locuteur choisit délibérément, proposant à son interlocuteur « de l'autre » à découvrir, ou bien, différemment, celui qu'il subit avec « l'autre » débusqué par le récepteur, et que celui-ci lui impose à son « intention défendante ».

\section{(i) Un risque choisi par l'énonciateur}

Ce risque " choisi », c'est, par rapport à « l'assurance » d'un X, dit $1 . .{ }^{45}$ marqué, celui - revers obligé des bénéfices de la connivence - des diverses réceptions malheureuses auxquelles se prête l'élément $\mathrm{X}$ correspondant, seulement donné à reconnaître :

- l'autre, reconnu comme autre par $\boldsymbol{R}$, lui demeure inconnu ; rien ne vient dans sa mémoire combler le « manque » qu'ouvre au fil du discours un indice perçu de discordance ou des guillemets de MA tendanciellement interprétés en MAE : c'est la très ordinaire et disphorique expérience de reconnaître que ce qui vous est donné à reconnaître - un discours autre spécifique - vous échappe ; comme par exemple, en (1), l'irritante énigme, pour un non familier de Proust, de cet ailleurs inconnu qui pourrait donner du sens à l'insolite évocation de ses heures de coucher par U. Eco, faute de quoi il éprouve qu'il demeure « en lisière » du sens, ou le sentiment en (24) de l'étrangeté de « cet Orient pas désert du tout » si sa protestation - marque de négation interprétable en indice dialogique - ne rencontre pas, dans la mémoire du lecteur, l'« ennui » d'Antiochus dans son « orient désert » ${ }^{46}$ :

(24) Le regard de Giraudoux va plus loin : "Les Fables de La Fontaine sont bien des contes, ce sont nos contes des Mille et Une Nuits » [...] Et voilà en deux mots la clef de La Fontaine, le plus oriental des classiques d'Occident. Il faudrait écrire un livre sur l'Orient et nous. [...] Et l'on découvrirait avec ravissement au sein de notre culture tout un royaume d'Orient. La Fontaine en serait le prince subtil et nonchalant [...]. Et voilà que s'accomplit le miracle : nous croyons à l'histoire qu'on nous raconte [...]. Et nous retrouvons, sans être désorientés, tout cet Orient pas désert du tout qui sommeillait en nous [C. Roy, La Conversation des Poètes, 1993].

Figure paradoxale mais courante, «l'allusion explicitée » met en œuvre la dualité spatiale du corps d'un texte et de ses notes pour " jouer sur les deux tableaux »,

45 L'incise est choisie ici comme marque de RDA neutralisant l'ensemble de oppositions entre modes (cf. chap. 9.3.3.2 p. 351).

46 « Dans l’Orient désert quel devint mon ennui! » [Racine, Bérénice, Acte1, sc. 4]. 
annulant le risque par la note sans renoncer à la connivence de l'implicite proposé par le texte ${ }^{47}$ :

(25) Comme si l'expérience venait moins « contredire $»^{2}$ la théorie [...] que la « négativer ». $/ 2$. Allusion au titre d'un texte de Freud : "Communication sur un cas de paranoïa venant contredire [mes italiques] la théorie psychanalytique » [J.-B. Pontalis, Perdre de vue, $1988: 73]$.

- l'autre n'est pas reconnu comme tel par $\boldsymbol{R}$; le Discours est reçu comme directement assumé par $\mathbf{L}$ : à manquer la RDA c'est, selon les cas, un déficit de sens ou un contre-sens qui se produit. Ainsi de la voix de F. Giroud en (2) amputée de la portée qui la double de son accent de défi pré-révolutionnaire ; ou de cette déclaration :

(26) Le 16 mars, la droite a remporté une victoire, mais elle n’a pas gagné la France. [J.P.Chevènement, discours du 20-04-1986, cité in Libération 21-04-1986]

qui, si l'écho des hauteurs visionnaires de la parole gaullienne où elle entendait se situer $^{48}$ est perdu, redescend vers l'ordinaire du commentaire électoral.

Un cas intéressant, au plan théorique, est fourni par l'analyse, dans le cadre polyphonique de la Scapoline ${ }^{49}$, d'un énoncé de Proust :

(27) Cependant les aéroplanes venaient s'insérer au milieu des constellations et on aurait pu se croire dans un autre hémisphère en effet, en voyant ces « étoiles nouvelles ». [Proust, Le Temps retrouvé]

Il est convoqué au cours d'une analyse des segments guillemetés dégageant 4 types de valeurs pour un « $\mathrm{X}$ », calculées à partir des seuls co-textes immédiats, internes à l'énoncé : elle oppose ainsi au cas (1) de « X » avec explicitation d'un « tiers individuel responsable de $\mathrm{X}$ », plaçant le locuteur de l'énoncé « dans une non-reponsabilité totale », du type "X » selon les paroles de Jean, celui (4) du « $\mathrm{X}$ » sans source explicite et attribué au ON polyphonique (« tiers collectif») être discursif aux contours flous qui, incluant $\mathbf{L}$, le place dans une «non-responsabilité partielle », cette attribution " particulièrement nette quand le pronom on est présent dans le co-texte » étant exemplifiée par l'énoncé (27). Or il suffit que, à l'appel à l'interprétation que constitue tout guillemet, réponde dans la mémoire ${ }^{50}$

47 Cf. Authier-Revuz (2002 : 289) et Lefèbvre (2004, 2007).

48 « La France a perdu une bataille! Mais la France n’a pas perdu la guerre! » [Appel du 18 juin 1940 (Affiche)].

49 (Fløttum 2001: 126 sq.) ou (Nølke et al. 2004 : 77-82).

50 Rencontre préparée, en amont immédiat, par la densité chez Proust (« mer, tempête, ciel, étoiles, pâles poussières d'astres, errantes voies lactées ») de la double isotopie mer/ciel étoilé qui 
le célèbre sonnet dédié par Hérédia aux « Conquérants » du nouveau monde, dont le tercet final fixe l'image de ces navigateurs fascinés par l'apparition des « étoiles nouvelles » de l'hémisphère austral

Ou penchés à l'avant de blanches caravelles /Ils regardaient monter en un ciel ignoré /Du fond de l'Océan des étoiles nouvelles.

pour que s'impose absolument le « tiers individuel responsable de X », d'un " comme a dit Hérédia » qui, interprétativement équivalent au cas (1), bouscule l'analyse proposée du couple co-textuel « $X » / o n$.

La visée de cette remarque n'est évidemment pas celle d'une critique - facile et absurde - d'une « lacune » de mémoire... car la mémoire « totale » apte à reconnaître « toutes » les allusions d'un texte est pur fantasme ${ }^{51}$ : le « défaut » d'adéquation de la mémoire réceptrice - que creuse inévitablement la différence des mémoires - est constitutif du sens qui, pour tous les sujets parlants, se produit dans et avec les aléas, les incertitudes du jeu mémoriel ${ }^{52}$. En revanche, ce que cet « incident interprétatif » met en évidence, c'est la limite de la « calculabilité » intralinguistique du sens telle que - à l'encontre du dialogisme bakhtinien et de l'interdiscursivité en $\mathrm{AD}^{53}$ - la conçoit l'approche polyphonique.

Si dans ces divers cas, l'autre discours perdu est seulement comme un « bateau de sens » en plus qui, faute d'une mémoire favorable, n'a pas pu atteindre le port, dans d'autres, la méconnaissance de la part d'autre que le Discours donnait à reconnaître en lui est source de véritable malentendu. C'est, bien entendu, le cas

traverse aussi le sonnet de Hérédia et qui donne sens à l'indice dialogique - autrement étrange $\mathrm{du}$ « en effet » répondant à cet autre discours où, de fait, les « étoiles nouvelles » marquaient bien l'arrivée des conquistadors dans « un autre hémisphère ».

51 Cf. A. Compagnon (2000) évoquant, illustré par des exemples pris chez Proust, d'allusions qui lui avaient échappé lors de son édition de Proust, le caractère de "cimetière des allusions perdues » que présente « la littérature » - et Proust tout particulièrement.

52 C'est comme une « extension de mémoire » que propose au lecteur ordinaire l'appareil éditorial de notes telles que « Écho de la célèbre expression... ; parodie des paroles de... ; citation d'une pantomime ...; Pastiche de Corneille... » dans une édition du Père Goriot (Garnier 2008, notes $8,17,55,74)$.

53 Rappelons le caractère central de l'opposition théorique, formulée par exemple par Pêcheux dans « Lecture et Mémoire » (repris in $(1990: 291)$ : « O. Ducrot se refuse absolument à faire intervenir dans l'analyse linguistique de la séquence la référence à quelque corpus interdiscursif que ce soit [...] » là où, pour l'AD la séquence ne peut être étudiée que si elle est mise en rapport avec l'interdiscours qui l'enveloppe, ou avec ce que Foucault (1969: 128) évoquait, pour tout énoncé, comme ses « marges peuplées d'autres énoncés ». Cf. aussi Adam (1999: 127) rappelant qu' « une analyse pragmatico-linguistique gagne à penser les signes de la langue de façon résolument bakhtinienne [...]. » 
lorsque le discours méconnu comme autre donné à reconnaître résonne dans le Discours - antiphrase, ironie - comme ce à quoi celui-ci s'oppose, l'énonciateur jouant alors « gros jeu » dans ce type de choix du risque : en relèvent le célèbre contre-sens sur la justification de l'esclavage des nègres par Montesquieu, les mésaventures connues d'humoristes (d)énonçant ironiquement des propos racistes ou antisémites ${ }^{54}$, applaudis par des spectateurs qui les prennent au pied de la lettre ou cet échange contradictoire à partir d'un bivocal méconnu :

(28) Marcel Bluwal : Je me souviens qu'en 68 [...] il a été mis à la poubelle un certain nombre de valeurs traditionnelles, et j'ai été frappé par le côté non dialectique de cette démarche. Elle était purement morale : les valeurs polluées par la bourgeoisie devaient être rejetées [...].

Bernard Pingaud: Maintenant, quand Bluwal parle des jeunes de 1968 qui voulaient mettre à la poubelle les valeurs polluées par la bourgeoisie et qu'il leur reproche une attitude antidialectique, j'ai bien envie de lui demander à mon tour s'il est très dialectique d'évoquer des valeurs éternelles, objectives, que tel groupe social aurait souillées, mais à qui nous pourrions, demain, rendre leur pureté originelle. Je ne crois pas que la conception marxiste admette de telles valeurs.

Marcel Bluwal : Je suis tout à fait d'accord avec Pingaud et je le dis tout de suite, d'autant plus que j’ai employé un langage entre guillemets qui n'était pas le mien [Morale et société, Semaine de la pensée marxiste, Ed. Sociales, 1974].

Ce sont aussi de vrais quiproquos à même de bloquer un échange que peut faire naître une RDA manquée : témoin cette conversation détendue entre un couple en vacances $(A, B)$ et un entrepreneur du crû (C) à propos de la vie locale, qu'une imprudente mise en jeu de mémoire discursive ${ }^{55}$ de la part de $B$ menace de faire capoter, dans un double malaise des « faces » des interlocuteurs :

(29) A - D’après ce que j’ai compris, il a vu trop grand, et puis il avait quasi tout misé sur un gros chantier qu'il n'a pas eu... alors, pour le moment, tous les beaux projets de l'an dernier sont à l'eau !

B - Adieu, veau, vache, cochon, couv/

C - Mais... il ne s'agissait pas du tout d'élevage, c'est un complexe sportif qu'il voulait.

54 Tel le sketch « Les vacances à Marrakech » (1975) que les auteurs (G. Bedos et S. Daumier) ont retiré de leur répertoire tant il suscitait d'approbations tranquillement racistes ; $c f$. aussi l'analyse par H. Godard (2011 : 253) du traitement par Céline dans Bagatelles pour un massacre du texte d'un auteur juif - qui « avait cru, en s'adressant aux antisémites pouvoir procéder par ironie »- qu'il reprend, à l'instar de « toutes les publications antisémites du moment » (et en omettant l'indice d'italiques du texte original) pour « faire dire [à celui-ci] le contraire de ce qu'il disait ». 55 Celle - très familière à A et $\mathrm{B}$, mais non à C - de Perrette (La Fontaine, Fables, VII 10) et de ses rêves de prospérité fermière « tombés » avec le « pot au lait » que, posé sur sa tête, elle portait au marché... Cet exemple - avec la gêne de B et la diversion opérée par A - est analysé de façon plus précise dans Authier-Revuz (2012). 
B - Oui, je sais..., c'était..., je pensais..., non, excusez-moi...

A (à C) - Et vous pensez qu'il y a une chance que ce soit repris ? C'était une bonne idée... [août 2008].

Remarque 2 : Prise de risque ou flottement de l'énonciateur. Je renvoie à la riche analyse proposée par B. Gardin et J. Richard-Zapella (1994) de la tempête politique au sommet du gouvernement allemand soulevée par le « risque » pris par le président du Bundestag le 10 novembre 1988, au cours d'une cérémonie commémorant la « Nuit de Cristal », à évoquer, à l'abri de la fragile distanciation d'un guillemet oral, l'opinion allemande de l'époque nazie : la précieuse revue des réactions (au-delà du naufrage initial de la cérémonie) dans la presse française fait apparaître le plus large éventail d'interprétations de ce discours, entre panégyrique du Führer prononcé sans le moindre « bémol » et " réquisitoire contre l'Hitlérisme » victime d'un flagrant contresens, en passant par la «stupéfiante maladresse », la « bévue » rhétorique « d'un usage fort malheureux de la citation », source de toutes les incertitudes que condense le titre de presse "L'incompris », pour en arriver au diagnostic d'un orateur « ventriloqué » par le discours nostalgique du $3^{e}$ Reich : « ça a parlé en lui », « il n’y a pas intention mais lapsus » - le risque « choisi » par l'inconscient est « subi » par l'énonciateur...

\section{(ii) Un risque subi par l'énonciateur}

Propice au ratage de l'autre donné à reconnaître, la possibilité du non-marquage peut en effet, à l'inverse, se prêter à des forçages interprétatifs, par $\mathbf{R}$, du dire de $\mathbf{L}$, lorsque, à l'encontre de l'intention de celui-ci, $\mathbf{R}$ identifie un élément de discours comme autre-donné-à-reconnaître dans un élément énoncé par $\mathbf{L}$, simplement - sans surplomb métalangagier -, comme « sien ».

On quitte ici le versant du risque « choisi », localement, par l'énonciateur lorsqu'il recourt à une forme non marquée de RDA, pour celui du risque « subi » par tout dire : reçu et compris dans la mémoire interdiscursive de $\mathbf{R}$, le dire de $\mathbf{L}$ est par là inévitablement " exposé » aux rencontres qui peuvent se produire entre les deux - énoncé de $\mathbf{L}$ et mémoire de $\mathbf{R}$ - et être interprétées par $\mathbf{R}$ comme RDA implicite.

Les incidents de frontière quant à ces discours autres que le dire donnerait - ou non - à reconnaître en lui peuvent, certes être empreints d'une flagrante mauvaise foi de la part d'un $\mathbf{L}$ protestant de son ignorance quant au dire autre que, sciemment, il fait jouer dans son dire : une forme non marquée de RDA apparaît alors comme stratégiquement choisie pour pouvoir toujours être déniée, en renvoyant $\mathbf{R}$ à des procès d'intention ou des délires interprétatifs ; c'est ce qu'illustre cet échange :

(30) J.M. Le Pen : « Monsieur Polac est un homme sans humour, sûr de lui et dominateur. [...Ils] présentent la politique et les valeurs fondamentales que je défends, comme une dérivation du nazisme [...]. » /Georges Kiejman, l'avocat des inculpés, interrompt : « Est-ce volontairement que vous employez les termes « sûr de lui » et « dominateur » que le général de Gaulle 
avait utilisés pour définir l'état d'Israël ? »/Jean-Marie Le Pen s'esclaffe : « Ça y est, voilà la notion d'antisémitisme introduite par le biais de De Gaulle. Non, je n'avais pas d'intention antisémite » [Procès en diffamation intenté par J.M. Le Pen à M. Polac et al., CR in Le Matin, 4.10.1984].

Mais l'affrontement ne sera pas moins vif lorsque c'est un élément énoncé, en toute « bonne foi » par l'un, comme « propre », que l'autre reçoit comme « autre » donné à reconnaître comme tel (RDA non marquée), c'est-à-dire en prêtant au premier une intention signifiante que celui-ci récuse... Tel est, par exemple, saisi par N. Sarraute, si attentive aux dangers que recèlent les incertitudes du dire, l'enjeu de la discussion pied à pied sur laquelle va se briser le mouvement qui portait deux amis, séparés par des choix de vie divergents, à se retrouver : celui du statut d'un « la vie est là » énoncé par $\mathrm{H}_{2}$, et où $\mathrm{H}_{1}$ entend - le recevant comme marque blessante de distinction " poétique » adressée au « béotien » qu'il pense incarner aux yeux de son ami - une allusion à Verlaine que récuse absolument $\mathrm{H}_{2}$ :

(31) $\mathrm{H}_{2}:[\ldots]$ Tu comprends pourquoi je tiens tant à cet endroit. [...]. $\mathrm{H}_{1}$ : Oui je comprends. $\mathrm{H}_{2}$ : $\mathrm{Si}$ je devais ne plus revoir ça, ce serait comme si, je ne sais pas moi, ... oui, pour moi, tu vois, la vie est là. [silence, puis léger ricanement de $\mathrm{H}_{1}$ ] $\mathrm{H}_{2}$ : Mais, qu'est-ce que tu as ? $\mathrm{H}_{1}$ : La vie est là, simple et tranquille... La vie est là, simple et tranquille, c'est de Verlaine, n'est-ce pas ? $\mathrm{H}_{2}$ : Oui, c'est de Verlaine, mais pourquoi $\mathrm{H}_{1}$ : De Verlaine, c'est ça ! $\mathrm{H}_{2}$ : Je n'ai pas pensé à Verlaine, j'ai dit la vie est là, c'est tout. $\mathrm{H}_{1}$ : Mais la suite venait d'elle-même, il n'y avait qu'à continuer. $\mathrm{H}_{2}$ : Je n'ai pas continué !... qu'est-ce que j’ai à me défendre comme ça, qu'est-ce qu'il y a ? qu'est-ce qui te prend ? [...]. $\mathrm{H}_{1}$ : Mais voyons, ne joue pas l'innocent... La vie est là, simple et tranquille. $\mathrm{H}_{2}$ : D'abord, je n'ai pas dit ça. $\mathrm{H}_{1}$ : $\mathrm{Si}$ tu l'as dit. Implicitement. Et ce n'est pas la première fois... [...] C'est là que tu te tiens, à l'abri de nos regards salissants, sous la protection des grands Verlaine. $\mathrm{H}_{2}$ : Je te répète que je n'ai pas pensé à Verlaine. $\mathrm{H}_{1}$ : Bon d'accord, admettons, je veux bien, mais tu reconnaîtras qu'avec le petit mur, le toit, le ciel par dessus le toit, on y était en plein. $\mathrm{H}_{2}$ : Où donc $\mathrm{H}_{1}$ : Mais voyons, dans le Poétique. La Poésie ! [...] [N. Sarraute, Pour un oui ou pour un non].

Ou bien cet échange conjugal où l'un $(\mathrm{A})$ reçoit un énoncé $\mathrm{P}$ comme forme non marquée de RDA, renvoyant polémiquement à sa propre parole - dont la forme marquée pourrait relever de « comme tu dirais » ou « tu dirais : " $\mathrm{P}$ " ", là où l'autre (B) proteste de la simplicité innocente de son dire de « $\mathrm{P}$ », dépourvu à ses yeux de toute malignité métalangagière :

(32) $A$ : Ce serait bien qu'on les invite un de ces jours. $B:$ Je vais y penser. $A:$ Tu te moques de moi ? $B:$ ?? $A$ : « Je vais y penser », tu me l'as assez dit, que c'est ma façon de botter en touche, non ? $B$ : [rire] C'est vrai... mais je n'y pensais pas du tout, j'ai dit ça parce qu'il faut vraiment voir comment, avec tout ce qu'on a à faire en ce moment... tu es pas un peu parano, mon grand ? Je n'avais aucune envie de t'agresser ! $A$ : Bon, bon, d'accord, si tu le dis... [conv. oct 2013]. 
La façon dont se « règle » le conflit sur le statut du discours autre dans ces énoncés fauteurs de trouble questionne la discrétude du tracé entre autre représenté et autre présent. Si les « bon d'accord » de $\mathrm{H}_{1} / A$ semblent mettre un terme à la discussion, les « admettons, mais tu reconnaîtras, si tu le dis » qui suivent indiquent que l'accord ne s'est pas fait pour autant sur le statut du dire en question dans le dire. Pour l'énonciateur $\mathrm{H}_{2} / B$, sa parole ne met pas en jeu de discours autre ; il évacue comme non signifiante ce qu'il juge une rencontre accidentelle, de hasard, entre ses mots et de l'ailleurs. Pour l'autre, s'il concède que ce qu'il a reçu comme RDA implicite puisse ne pas être le fruit d'une claire intention de l'énonciateur, ce n'est pas, à l'instar de celui-ci, pour le congédier hors du dire, comme un hasard, non pertinent pour le sens et n'impliquant pas le sujet du dire : il ne renonce pas à la signifiante présence, dans le dire du sujet, d'un ailleurs qui, sur le mode d'un « comme par hasard », réminiscence non consciente - ou voix secrète mais résolue de son inconscient - s'y ferait entendre...

Dans le cadre, différent, d'une lecture théorique, c'est un jeu de cet ordre que décrit J. Bres: celui d'une oreille qui, particulièrement sensible à un certain discours (Guillaume) ne peut manquer d'en percevoir la présence, comme refoulée, dans le dire d'un autre (Benveniste) :

(33) [...] on sait que Benveniste a pris grand soin à ne pas se référer à la théorisation de Guillaume [...], à (essayer de) gommer de ses textes toute trace d'intertextualité avec la psychomécanique. Peine perdue : chassé par la porte du discours rapporté, le discours de l'autre revient, par exemple par la fenêtre de la négation : « Du signe à la phrase il n’y a pas transition [...]» (1969/1974, p. 65, ibid.). Une oreille avertie en psychomécanique ne peut manquer d'entendre dans transition un mot de Guillaume dans son questionnement de la transition de la langue au discours. [1999: 83, idt, gras de mon fait].

Les « turbulences » aux frontières observées aux « bords » incertains de la RDA dans le Dire témoignent que ce n'est pas au même niveau que se situe ce qui est en jeu pour le sujet parlant dans ce tracé de frontière, selon que le ratage interlocutif relève du risque choisi ou subi par l'énonciateur. Si l'énonciateur peut se dépiter de ce que l'interlocuteur - manquant une allusion par exemple, et, avec elle, telle facette de l'image qu'il compose de son discours - le prenne, méconnaissant quelque chose de sa spécificité, en quelque sorte, " pour un autre », voire déplorer un blocage communicationnel, comme en (29), il s'agit de bien autre chose, pour lui, lorsque quelque chose de sa parole, énoncée comme propre, lui est retourné comme pur « écho » de déjà-dit : colère, protestation, trouble malaise... signifient que c'est alors (bien plus profondément que pour telle facette de son positionnement discursif) son statut de sujet parlant, source de «sa » parole qui se trouve, ponctuellement, mis en cause.

Ainsi, dans cet échange (à l'issue disphorique), la convocation par $\mathbf{R}$ du déjàdit habitant selon lui, le dire de $\mathbf{L}$, apparaît comme faisant violence à l'énoncia- 
tion « une » de son interlocuteur, ramené au statut de perroquet-porte-voix, en lui déniant son statut d'énonciateur d'une parole propre (de fait toujours susceptible de « faire un » avec de l'extérieur sans être nécessairement « ventriloqué ») :

(34) - Alors, qu'est-ce qui se passe à l'hôpital ? - Ils sont en train de détruire le service public de santé, de mettre en place une santé à deux vitesses, pour les riches et pour les pauvres. - Oh ! écoute, c'est pas tes tracts que je te demande, c'est ton expérience à toi, qui aimes ton boulot, pourquoi tu penses que ça va mal... [conversation entre deux amies dont l'une est infirmière, après des manifestations, avril 2009].

C'est l'intime violence de ce mécanisme qui, réduisant le dire de l'autre à une récitation, le dépouille de sa parole propre, que met en scène, poussé à l'extrême, la joute oratoire, brillamment présentée par A. Compagnon (1979 : 366), opposant dans Trans-Atlantique W. Gombrowicz, le narrateur, à Borgès : à aucun moment celui-ci ne répond aux propos de son adversaire, il se contente de les réduire au statut d'écho d'un déjà-dit dont, redoutable érudit, il déniche les références, jusqu'au « merde, merde, merde » de Gombrowicz exaspéré, encore une fois " annulé », en écho de Cambronne : cruel et virtuose, ce jeu est une mise à mort de la parole de l'autre, débouté de son statut d'énonciateur source de « sa parole », dépossédé de ses mots.

Dans tous ces conflits relevant $d u$ « risque subi », le message adressé à l'énonciateur est celui d'un « soit, cet autre discours n'est peut-être pas représenté dans ton dire, mais, de toute façon, ton dire le «présente ». L'ambiguïté du verbe « présenter » entre valeur passive (a) et active (b), comme dans

(a) le mur présente une fissure

vs

(b) l'enfant présente sa plaie au médecin le fermier présente ses légumes sur l'étalage

ouvre sur l'espace d'un autre présent(é) (a-b) où, en l'absence de seuils tranchés, c'est sans solution de continuité que - en deçà du seuil de la représentation - on passe d'un autre présent(é), au sens (a), à un autre qui, affleurant à des degrés divers à la conscience de l'énonciateur, peut être dit « présenté », au sens (b), par le dire. La vivacité des débats autour du statut de cet autre - représenté/présent(é)/ absent - dans le dire de l'énonciateur $\left(\mathrm{H}_{2}\right.$, B en (31), (32)) témoigne de la violence interprétative subie par celui-ci de la part de l'interlocuteur : celle-ci relève - que ce soit dans l'interprétation initiale d'un ailleurs représenté, intentionnellement et implicitement, ou, en repli, dans l'insistance sur le caractère néanmoins présent(é) de cet ailleurs dans le dire - d'une mise en cause, intrusive, des frontières dans lesquelles, pour l'énonciateur, était enclose sa parole « propre ». 
Remarque 3 : « Réminiscences anticipées ». Notons encore, relevant du « risque subi » par un dire livré à la réception interprétative du récepteur et de sa mémoire, le cas particulier de l'allusion perçue « illégitimement » relativement, non seulement à l'intentionnalité de l'énonciateur, mais même à la mémoire susceptible de lui être prêtée : celui du récepteur percevant - par erreur ou en assumant l'arbitraire d'un trajet intertextuel n'ayant de réalité que subjective, dans sa lecture - dans un texte l'écho d'un « déjà-dit » postérieur au texte. Tel le mécanisme (rappelé par Compagnon, 2009 : 26-28) des « réminiscences anticipées » qu'évoque Proust, dans une mémoire densément sédimentée de littérature, où peut surgir « une phrase de Flaubert dans Montesquieu »- mécanisme qui, de façon non consciente est évidemment à l'œuvre dans la lecture «naturellement anachronique » que nous faisons des textes dans notre mémoire.

Au-delà de sa pertinence comme concept à mettre en œuvre dans l'analyse sémantique des discours, l'extériorité interne au discours - ou son hétérogénéité constitutive, $c f$. chap. 10 - apparaît là, dans sa dimension d'expérience subjective foncière : celle - largement non consciente pour un sujet d'avoir à « se poser » dans le langage, à y assurer sa parole comme telle, imaginairement et vitalement soustraite à son ailleurs dépossédant - enjeu subjectif du tracé configurateur dont on envisagera, au chap. 13, les conditions et les aléas.

\subsection{2 Échos rencontrés dans le dire d'un autre : incertitudes et aléas}

En deçà des accidents perturbant, parfois vivement, le cours des échanges, c'est souvent à bas bruit que le récepteur fait l'expérience de son incertitude - émergence de la non-coïncidence foncière où se produit le sens - à reconnaître un fait de RDA non marqué, c'est-à-dire (1) à percevoir sur la chaîne l'écho d'un discours autre, (2) à identifier celui-ci, (3) à attribuer cet écho à une intention de l'énonciateur. De l'impression fugitive d'un " ça me rappelle quelque chose " ... à l'interrogation persistant sans réponse, le flottement de la réception du déjà-dit dans le dire - entre représentation et présentation/présence - prend de multiples visages.

Ainsi, si c'est sans doute aucun que tel récepteur de l'énoncé (1) de U. Eco peut transformer en allusion la « rencontre » opérée dans sa mémoire avec l'incipit de La Recherche, le degré de certitude est moindre pour les mots par lesquels J. Derrida ouvre sa conférence au colloque «Judéité - Questions pour Jacques Derrida ${ }^{56}$ :

(35) Longtemps et de bonne heure j’ai tremblé, je tremble encore devant le titre de ces journées [...] et jamais le privilège d'un colloque apparemment à mon adresse ne m’a à ce point intimidé, inquiété [...] [cité in Peeters (2010 : 613)].

Pour un lecteur privé de l'intonation du philosophe, l'écho, délibérément fragile, $\mathrm{du}$ « longtemps ... de bonne heure » peut demeurer indécis. Mais, quoiqu'il en soit, la lecture commune poursuit normalement son chemin au fil des perplexités dont

56 Cf. colloque 3-5 décembre 2000 à Paris, publié sous ce titre, Cohen J. et al. (dir.) 2003. 
elle s'accommode avec plus ou moins de cet « inconfort de lecture » qu'évoque P. Hamon (2000 : 185) dans ses réflexions et ses exemples ${ }^{57}$ sur le " plus ou moins de certitude » éprouvée quant à l'identification d'une « allusion » - le degré d'inconfort étant fonction de l'inscription plus ou moins intense du lecteur dans une perspective d'accès à la « vérité » d'un texte. Et, devant la « légion de références et emprunts » que lui-même et d'autres ont « cru déceler » dans un texte de Spinoza, P. Zaoui ${ }^{58}$ dit son doute

qu'aucun de ces rapprochements vaille en lui-même ou puisse valoir [pour Spinoza] comme argument d'autorité (Comment savoir pour chacune si l'allusion est délibérée ou non ?) [...].

pour ne retenir, comme pertinent pour la pensée et l'écriture de Spinoza que le fait de son intense inscription dans le déjà-dit.

Dans le contexte, différent, des discours médiatiques à forte « circularité des mots et des dires $~^{59}$, tissés de RDA inégalement marquées, les analyses montrent l'importance des « reprises » - non marquées comme telles - inassignables de façon discrète à du représenté ou à du présent(é).

Ainsi de l'abandon des guillemets observé par S. Moirand ${ }^{60}$ dans l'emploi, par exemple, de " contaminé " lors de l'affaire, en mai 2000, du colza transgénique : au fil du déroulement des articles d'un même numéro du Monde la métaphore de la « contamination » d'abord explicitement empruntée aux écologistes fait place à des apparitions, sans marquage, susceptible de fonctionner sans solution de continuité - brouillant le tracé du contour de l'hétérogénéité représentée dans l'hétérogénéité constitutive - comme écho prolongeant la distanciation des formes marquées de l'emprunt ou (ayant rompu les amarres avec leur « source») comme intégré à la parole propre. De la même façon, D. Da Cunha (2012), en écho à l'insistance de Bakhtine sur la « gradation infinie sur les degrés de l'altérité (ou

57 Déclinés en « je ne suis pas très sûr que dans [...], et malgré les notes de telle édition critique il y a allusion [...]. [...] je ne suis pas très sûr (mais quand même un peu plus [...]) que [...] est une allusion à [...]. En revanche, je crois identifier avec certitude une allusion assez nette au [...]. [...] Mais comment évaluer ces degrés? » [Hamon (2000: 185), idt]. De cette incertitude témoignent les « peut-être » qui, régulièrement, modulent en hypothèses les élucidations d'échos dans les éditions de textes, tel ce « peut-être » : « peut-être [Balzac] se souvient-il de la comédie vaudeville en un acte [...] » (note 95 dans l'édition du Père Goriot évoquée ci-dessus note 53), comme aussi les reproches d'arbitraire ou de surinterprétation que suscitent certains « dévoilements » d'allusions.

58 Pierre Zaoui, Spinoza. La décision de soi, Paris, Bayard, 2008.

59 Moirand (2007: 158).

60 Moirand (2007 : 47-50). Le même chemin, allant d'un « X » comme emprunt marqué à un X intégré au fil du dire, est illustré dans cette étude par de nombreux cas: « principe de précaution », « vache folle », ...). Cf. aussi Moirand (2006). 
de l'assimilation) du mot » dans le dire, comme de la conscience qu'en ont les énonciateurs ${ }^{61}$, fait apparaître dans la circulation des dires propres à un "Courrier des lecteurs ${ }^{62}$, en deçà des formes marquées de RDA, la zone indécise où « il n'est pas facile de distinguer entre allusion et discours autre présent (et non représenté) ».

Dans tous ces cas, un élément dans un dire est entré en résonance avec la mémoire d'un récepteur : c'est la conversion interprétative, en forme de RDA non marquée, d'un fait de concordance avec de l'ailleurs, qui se charge d'incertitude.

Mais c'est aussi le fait même que la rencontre se produise entre un dire et une mémoire vivante donnée qui peut se révéler comme aléatoire, et la perception subjective d'un « même » - contrairement aux résultats automatiques de la mise en rapport en $\mathrm{AD}$ entre un texte et une « mémoire » interdiscursive constituée en corpus ( $c f$. ci-dessus, chap. 10) - comme imprévisible.

Revenant sur l'impossible "dépliement " en note par un éditeur de La Recherche de toutes les allusions que le texte recèle, Compagnon (2000) évoque, par exemple, telle allusion à des vers - pourtant bien connus de lui - de Baudelaire qui, lui ayant « échappé » au moment de l'édition, s’impose à lui, dans un autre temps, comme " immanquable », lui suggérant ce commentaire : « Je m’en veux [...] de n'avoir pas vu l'évidence $»^{63}$.

J’ai, pour ma part le souvenir étonné de ces « intermittences de la mémoire », jouant dans la rencontre d'un " même » - redoublé de l'incertitude à interpréter le statut de ce même pour son énonciateur : ainsi ai-je plusieurs fois étudié, avec des étudiants, un texte de Gide, comportant

(36) M. Granville, journalier, a été attaqué à une heure du matin, rue Barbet, à Rouen, par un malandrin [...]. La victime se déclare incapable de reconnaître son agresseur ; mais, à ses cris, Mme Ridel avait mis le nez à la fenêtre et prétend avoir pu reconnaître en lui le sieur Valentin, journalier, qui comparaît à présent devant nous. [A. Gide, Souvenirs de la Cour d'Assises, VI, Pléiade, p. 645]

sans y entendre d'écho... jusqu'à ce que - pour une raison que j'ignore - se soit soudain éveillé le vers qui m'était depuis longtemps familier «La Belette avait mis le nez à la fenêtre », surimposant, désormais pour moi, à cette Mme Ridel sûre

61 Bakhtine (1984 : 330), cf. aussi « Notre parole [...] est remplie des mots d'autrui caractérisés, à des degrés variables, par l'altérité ou l'assimilation, caractérisés, à des degrés variables également, par un emploi conscient et démarqué » (ibid : 296), cités par Da Cunha (2012).

62 Corpus d'une soixantaine de lettres, publiées dans des quotidiens brésiliens sur une courte période (oct.-nov. 2005) en réponse au reportage d'un magazine alléguant un financement secret par Cuba de la campagne présidentielle de Lula.

63 Compagnon (2000: 244-245). 
d'elle et prompte à charger un suspect, la figure de l'arrogante et sans scrupule « Dame au nez pointu » croquée par La Fontaine dans « Le chat, la belette et le petit lapin » (Fables, VII 16) ; et à la demande adressée à un collègue, visant à évaluer la « légitimité » d'un écho, pour moi aussi soudain que têtu, la réponse d'un évasif " pourquoi pas ? », n’a fait qu'ajouter à l'expérience des caprices de la mémoire, quant à cette perception d'un même, celle du flottement de son attribution à un emprunt volontaire, ou à une réminiscence... chez Gide amateur de La Fontaine, plutôt qu'au hasard, non éliminable, des similitudes !

\subsection{3 Échos rencontrés dans son propre dire : après-coups, flottements, surprises.}

$\mathrm{Au}$ nombre des expériences de flottement dans la rencontre et l'interprétation d'un déjà-dit du dire, il faut ajouter, troublante, celle que fait l'énonciateur luimême dans son propre dire, découvrant, sous de multiples facettes, combien il n'est pas le maître lucide des échos que « ses mots » portent avec eux - trivialement : combien il ne sait pas très bien... ce qu'il dit.

Ce peut être, dans un après coup de l'écriture, qu'un énonciateur reconnaît le caractère d'emprunt d'un élément, accueillant, avec ou sans plaisir, dans son dire la mémoire interdiscursive singulière attachée à cet élément, qu'il avait mise en jeu à son insu !

Ce retour de l'énonciateur sur son dire et les échos qui s'y révèlent peut se faire en réponse aux remarques du récepteur dont - contrairement aux réactions évoquées plus haut - l'énonciateur admet le bien-fondé : c'est ainsi le cas d'une auto-critique, parue à la suite de protestations de lecteurs, du journal Le Monde (15.10.2001) ${ }^{64}$ reconnaissant la présence non réfléchie d'un déjà-dit inopportun, dans l'emploi du terme « jeunes » comme désignation « euphémique » - promue par la presse d'extrême droite - de jeunes maghrébins, noirs, immigrés.

Sans l'intervention d'un autre, c'est aussi l'énonciateur lui-même, mais dans l'écart à lui-même d'un autre temps, qui perçoit- sur les modes les plus divers un déjà-dit qu'il n'avait pas délibérément mis en jeu : ainsi, de cette « remontée » dans la mémoire à partir de mots venus simplement - « sans histoire » pourrait-on dire - sous sa plume, dont A. Compagnon (2013) retrace, précisément, à la façon d'une enquête, les péripéties :

64 Étudié par F. Hailon (2012a), dans le cadre d'analyses précises de l'emprise dans la presse des « représentations du FN » qui y apparaissent, « en surplomb comme déterminantes » (p. 196) (cf. aussi 2011, 2012b). 
(37) [...] dans La classe de rhéto, pour décrire l'humeur de l'un de mes camarades, mon meilleur ami, qui était un peu fou, j'ai écrit [1] qu'« il passait sans transition de l'exaltation à l'abattement ». Puis il y a quelques jours, je me suis soudain dit [2] que cette phrase qui me trottait dans la tête depuis quarante-cinq ans n'était pas de moi mais devait être une citation que j'avais lue à l'époque qui m'avait fait comprendre le comportement de mon ami en le nommant. [...] Cette phrase, je l'avais lue quelque part [3] en 1965, elle avait soudain éclairci pour moi le comportement de ce garçon ; elle était restée inscrite dans ma mémoire et je l'ai finalement écrite. Et comme, aujourd'hui, on peut tout retrouver grâce à Google, je l'ai tapée [5] [...] et maintenant je sais [6] que je l'avais lue dans le Lagarde et Michard du XVIIIe siècle, à propos de Diderot, non de Rousseau, comme je me le suis d'abord dit [4] en le retrouvant l'autre jour [2013 : 24, chap. « Une jeunesse liseuse »].

Dans les parcours génétiques, c'est tout un pan des rectifications qui relève de la rencontre, à la relecture, non pas de répétitions malencontreuses, de sonorités fâcheuses, de clarté douteuse, etc... mais, dans les mots choisis tout d'abord - naturellement - d'un déjà-dit éprouvé comme inopportun. Ainsi G. Philippe (2012 : 258 sq.) analysant ce qui se joue dans les variantes paraphrastiques observables dans le manuscrit « Cuba » de J.P. Sartre, fait-il apparaître une série de suppressions, à la relecture, de termes conformes à " l'ethos prédiscursif [d'] intellectuel engagé qui décrit le monde à travers un ensemble de catégories idéologiques » du premier jet, au profit du vocabulaire " moins marqué idéologiquement » requis par la visée « littéraire » d'un idéal de « belle langue » : surexploités, structure, aliénation, remplacés respectivement par pauvres, cause, violence.

Ce peut être aussi, dans le mouvement même du dire, dont il est, à tout moment l'auto-récepteur, que l'énonciateur perçoit une résonance interdiscursive non programmée qu'il peut accueillir comme bienvenue :

(38) En ce temps là (voilà que je me mets à parler comme un apôtre quand je repense à Mao, l'autre saint patron de ma jeunesse folle au deux sens de ces mots), j'avais vingt et quelques ans [...], et je faisais tous les matins mon chemin de croix. J'allais à la recherche du prolétariat immigré des usines [M. Schneider, « D’une passion l'autre », in Lacan, les années fauves : PUF, Paris, 2010, p. 4].

ou reconnaître avec irritation, sous l'expression affective la plus spontanée, la mécanique ventriloquée qui la porte :

(39) C’est vraiment embêtant. Mais je n'ai qu'à m’en prendre à moi-même... Merde alors ! C'est ma mère qui me disait ça... Si je me mets à parler comme elle ! J'avais horreur de ça ! [conversation, 02-2010].

Ce mouvement de retour " désappropriant » sur son propre dire, se trouve multiplement illustré dans un roman qui en fait un trait des monologues intérieurs d'un personnage, comme par exemple dans : 
(40) (a) Oh, mon Dieu, elle devenait fasciste en vieillissant. Elle n’allait pas tarder à réclamer le rétablissement de la pendaison et de la flagellation, enfin peut-être pas de la peine de mort - après tout pourquoi pas ? [...] des saligauds qui torturaient enfants et animaux et machettaient des innocents. "Saligauds " c'était un mot typique des tabloïds, sorti tout droit du Sun [...]. Si ça continuait, elle ferait aussi bien d'annuler tout de suite son abonnement au Guardian.

(b) Amelia avait eu, ce soir, une conduite encore plus bizarre que d'habitude : elle avait débité des âneries au sujet d'Olivia alors que [...]. Débiter des âneries. Encore une expression de son père. Ça faisait presque un an que le vieil homme était mort [K. Atkinson, $L a$ Souris bleue, trad. fr. Le Livre de Poche, 2006].

C'est, enfin, de ce mouvement que relève la troublante surprise du « lapsus interdiscursif » par lequel c'est à travers le déjà-dit attaché à une formulation (un des environnements discursifs où elle a vécu un épisode marquant de sa « vie de mot », pour parler comme Bakhtine), et non par le classique mot pour un autre, que l'inconscient trouve à se dire sans le concours ou à l'encontre de l'intentionnalité : ainsi, cet énonciateur évoquant, entre amis, la cure psychanalytique qu'il a entreprise

(41) Bof, si mon analyse se poursuit fraîche et joyeuse, normalement ça devrait aller [Oral, nov. 1990].

qui adhère, avec un rire, dans le second temps d'une auto-réception d'abord déconcertée, à ce que, récepteur de ses propres mots, il y a entendu, à savoir que, selon lui, la psychanalyse a, pour lui, quelque chose d'une guerre ${ }^{65}$.

Ou bien, dans une formulation où s'impose un déjà-dit aussi impérieusement survenu qu'il est malvenu - lors d'un oral de concours, où une candidate, mise en difficulté par le jury au sujet de son interprétation de La Route des Flandres de C. Simon comme porteuse d'un « message progressiste » appelant à une « transformation du monde ", et qui, désireuse de concéder, en dépit de sa conviction spontanée, un certain pessimisme dans la vision de l'auteur, emprunte - sans en avoir conscience dans un premier temps - les paroles de... l'Internationale appelant à faire « du passé table rase », qui, adressées à ces interlocuteurs, lui reviennent en boomerang, avec leur « suite » dans le déjà-dit : « ... debout ! debout ! Le monde va changer de base, etc. »

(42) C'est vrai que Claude Simon fait du passé table rase ... enfin ce n'est peut-être pas le mot, il ne reste pas grand chose de solide [Oral de concours de recrutement d'enseignants de lettres, 1998].

65 « La guerre fraîche et joyeuse » est une boutade du Kronprinz Guillaume de Prusse en 1914. 


\section{Hors RDA : l'ailleurs discursif consciemment « fait sien » dans le dire}

Ainsi - avec des turbulences observables sur ses « marges » non marquées -, la RDA dessine-t-elle, dans un discours que traverse de part en part une extériorité discursive $\boldsymbol{E}$ illimitée, la place $\boldsymbol{E}$ ' de ce que ce discours désigne en lui comme discours autre. Sur cette base, il convient de ne pas passer à une géographie trop simple des jeux de l'ailleurs discursif dans le dire, où à la partition $\boldsymbol{E} / \boldsymbol{E}$ se superposerait l'opposition conscient/non conscient.

Certes, la RDA implique l'intentionnalité ; et, solidairement, l'ignorance - la non conscience - pour l'énonciateur du déjà-dit traversant constitutivement son dire, est condition de la possibilité même de ce dire, qui ne peut «se tenir » que protégé ( $c f$. chap. 13, 14 ci-dessous) des forces centrifuges, dépossédantes, qui le « déferaient ». Et, envisagé par le dialogisme, le caractère insu du déjà-dit travaillant le dire est au cœur même du projet de l'AD, comme continent à explorer nécessairement pour approcher le "sens », et s'impose comme facteur pertinent dans toute rencontre - perception ou analyse - de l'espace stéréotypique des lieux communs, clichés, routines, formules... qui viennent d'autant plus naturellement aux énonciateurs qu'ils les ont « reçus » d'ailleurs sans le savoir.

Pour autant, apparaît en $\boldsymbol{E}$, hors RDA, une zone où c'est en toute conscience de l'énonciateur que du déjà-dit est mis en œuvre, dans son dire, comme - approprié, adopté, assimilé... - délibérément fait sien. Son existence apparaît - sans être thématisée - comme l'un des pôles du continuum des " plus ou moins conscient ", "plus ou moins réfléchi » souvent évoqué à propos du fonctionnement énonciatif de la stéréotypie ${ }^{66}$ tel qu'il s'observe massivement dans les genres « routiniers » $(v s \text { « auctoriaux », cf. Maingueneau 2004) })^{67}$.

Le souci d'identifier, dans l'ensemble des modalités sur lesquelles l'extériorité constitutive de tout dire joue en lui, celle d'un déjà-dit simplement conscient - donc pas insu, sans être représenté pour autant - se fait jour, clairement expli-

66 Cf. par exemple Branca (1993) analysant le routinier dans « l'écrit des assistantes sociales » comme relevant de «fonctionnements qui sont des savoir faire plus ou moins conscients »; ou Amossy (2010) notant que c'est “sciemment ou non”, « de façon plus ou moins réfléchie et apprise », que l'expression dans des genres fortement codés se régule, stéréotypiquement, selon un « modèle préexistant » (p. 50-51).

$67 \mathrm{Si}$, à l'interrogation de P. Veyne (1995 : 208) « Les lettres administratives qu'écrit Stendhal quand il est commissaire des guerres, font-elles partie de ses œuvres ? Est-ce qu'il en est l'auteur ? » il est possible de répondre de façon négative, Henri Beyle n'en est pas moins leur énonciateur, adoptant, sans doute, selon toute la gamme du « plus ou moins conscient », les normes de cette correspondance. 
cité, chez F. Sitri dans un texte (2015a) revenant sur l'ensemble de son parcours en $\mathrm{AD}^{68}$. Menant une réflexion exigeante sur les divers aspects de l'extériorité discursive interne à un discours, à travers le traitement minutieux de corpus d'écrits professionnels, elle pose comme « une question importante [...] celle du caractère conscient ou non des routines » (p. 176, 182). Formulant - pertinemment - son interrogation d'ensemble en termes d'un « même » identifiable, sans marquage, entre un discours et de l'extériorité discursive, et recouvrant des phénomènes non équivalents, elle propose de distinguer entre les mêmes

(1) du répété (non conscient) vs de la reprise (consciente),

(2) de la reprise d'emprunt (RDA d'allusion) vs du partage (routine assumée).

Ce mode d'accueil conscient du déjà-dit dans le dire n'empiète nullement sur la zone $\boldsymbol{E}$ ' de la RDA : c'est du complémentaire de $\boldsymbol{E}$ ' dans $\boldsymbol{E}$ - la part d'ailleurs discursif jouant dans le dire sans y être représenté - qu'il relève, partageant de façon non discrète cette zone du déjà dit présent(é), selon que ce déjà-dit « fait sien » est énoncé comme tel consciemment ou non par l'énonciateur.

Aussi, n'est-ce pas en tant que tel que ce troisième mode de tissage du déjà-dit dans le dire - ni insu, ni représenté - intéresse une étude de la RDA, mais en ce que leur base commune - la conscience de la mise en œuvre d'un ailleurs discursif dans le discours en train de se faire - permet, différentiellement, d'affiner ce qui fait la spécificité de l'une, la RDA - une fonction dissimilatrice, incompatible avec le mouvement sous-jacent, consciemment assimilateur de l'autre.

Remarque 4 : Présence multiforme de modèles consciemment suivis. De multiples observations témoignent de la réalité de la pratique du dire se conformant - en toute conscience et sans le dire - à un « modèle » préexistant. Il en va ainsi, lors des processus d'apprentissage, des pratiques écrites ou orales relevant de la formation professionnelle, avant que les modèles extérieurs ne soient éventuellement incorporés en "seconde nature »; du nombre et de la diffusion des ouvrages pourvoyant les énonciateurs en instructions ou en modèles : manuels à l'usage des professionnels de la rédaction (comptes rendus officiels, textes à caractère juridique...), guides pour le courrier administratif, recueil de lettres et discours pour toutes les circonstances de la vie sociale et privée... ; d'expériences parfois assez disphoriques à devoir faire passer son dire par les filières très normées des formules d'appréciations de bulletins scolaires, de lettres de recommandation ou de témoignages de voisinage... ; des « éléments de langage » que les acteurs de la vie politico-médiatique vont répétant à l'identique, successivement ou simultanément à travers plateaux et studios les soirs de résultats électoraux sur le mode de l'expression, hic et nunc, de leur pensée personnelle ; de tous les rituels, enfin - civils ou religieux - dont l'accomplissement passe par la ré-énonciation, à neuf, avec sa valeur performative, d'un énoncé strictement figé : des « je vous déclare unis par les liens du mariage » ou « la séance et ouverte » aux paroles des

68 Notamment dans le cadre du « travail social » (rapports éducatifs, signalements d'enfants en danger...) : cf. par exemple, en collaboration, Cislaru et al. (2008), (2013) ou Née et al. (2014, 2016). 
fidèles et de l'officiant de la messe, ou au serment publiquement énoncé comme répétition du nouveau président des Etats-Unis.

Il n'est pas question ici de rendre compte, même sommairement, du champ passionnant et difficile - en bordure externe de la RDA - de ces dires consciemment coulés dans un moule préexistant ; ni d'en évoquer l'importance dans le fonctionnement social, de dégager les profondes différences qui le parcourent au plan énonciatif (statut du « modèle », opposé, par exemple, dans l'effectuation du dire des « éléments de langage » ou de celui des rituels sacralisés qui requièrent sa dissimulation pour les premiers et sa pleine reconnaissance pour les seconds), ni d'en interroger l'extension (relativement, notamment, au statut, aussi complexe que débattu, de la « récitation », ou de la « parole du comédien »...). Je noterai seulement, dans le champ des « prêts à écrire », l'intérêt des approches dégageant, au-delà de l'image négative d'une « communication bloquée », d'une " parole mortifiée », « vidée de sens » et « d'authenticité », le double versant positif du « modèle » dont « s'emparent » - plus qu'il ne s'impose à eux, les privant de parole - les énonciateurs peu lettrés comme de « facilitateurs d'accès » à une expression écrite personnelle, et qui constitue une manifestation d'appartenance à un groupe ${ }^{69}$.

Le discours autre « fait sien » n'est pas « de la RDA non marquée » : non seulement le mouvement énonciatif conscient de « faire sien » du dit « ailleurs-avant-et indépendamment » ne se confond pas avec celui de la RDA qui, au contraire, désigne « de l'autre » dans le dire, mais ces deux rapports du dire au déjà-dit s'opposent et s'excluent.

Dans le mouvement par lequel un dire passe consciemment par les filières d'un déjà-dit - celui de l'assimilation d'un ailleurs avec lequel il ne fait plus qu'un - la moindre manifestation dans le dire d'une prise de distance différenciatrice vis à vis de cet ailleurs est radicalement « subversive ». Glisser, dans la reprise d'une formule consacrée, des guillemets (de MAE), un etc. indiquant que le degré de familiarité avec le déjà-dit permet de se dispenser de le dire effectivement ${ }^{70}$, des (!), (!!) de distanciation méta-énonciative, a fortiori des commentaires explicitant les comme on dit, comme il est coutume de dire - que, précisément, résorbe le mouvement $d u$ " faire un » - c'est-à-dire insérer le moindre grain de RDA, c'est y introduire le « diable » d'une distance dissimilatrice, provocatrice. Il en est ainsi, par exemple, de l'intonation de « perroquet » faisant entendre que

69 Cf. outre Branca (1980 : 24), les précieux travaux de cet auteur consacrés à l'écriture des « peu lettrés », notamment (2014) au vaste corpus de 700 lettres de soldats de la grande guerre qui, prenant la plume pour une correspondance à forte charge affective, alors qu'ils « n'écrivaient jamais dans la vie », trouvent une aide dans les « cadres » fournis par les répétitives formules de début et de fin de lettre ; $c f$. aussi Sitri $(2015: 174)$ soulignant la nécessité de « remettre en question l'association entre "routine" et "pure répétition" ".

70 Tels ceux évoqués ci-dessus, chap. 4 ex. (47), (48), p. 123, comme rappels de la présence de $\mathbf{L}$ dans la représentation qu'il énonce, au DD, d'un dire de $\boldsymbol{l}$. 
l'énonciateur « déshabite » un dire qu'il ne « fait pas sien », dans cet échange sur lequel s'achève l'affrontement d'un enseignant et d'une élève :

(43) - Répète après moi : monsieur, je m’excuse d'avoir été insolente envers vous.

- Je n'ai pas été insolente.

- J'attends : monsieur, je m'excuse d'avoir été insolente envers vous.

- Monsieur, je m'excuse d'avoir été insolente envers vous.

C'était récité mécaniquement, avec une ostensible absence de conviction. J'ai quand même tendu le carnet qu'elle a aussitôt saisi avant de sautiller vers la porte. Au moment de disparaître dans le couloir, elle s'est exclamée

- j’le pense pas.

J'ai bondi mais trop tard [F. Bégaudeau, Entre les murs, 2005, p. 56].

de formules dont la « politesse » serait annulée par la désinvolture de leur renvoi à du stéréotype par etc. ou des points de suspension :

(44) Je vous prie de croire etc.

Veuillez agréer l'expression...

comme de paroles consacrées dont - énoncées par un « officiant » lassé de leur répétition - s'évanouirait la valeur performative :

(45) Je vous déclare unis et cotera...

Et au scandale - « profanation pure et simple » écrivait M. Droit dans le Figaro Magazine du 01-06-1979 - causé par le succès de la version « reggae » de l'hymne national par S. Gainsbourg « Aux armes et cætera », répond le mode irrévérencieux sur lequel le pittoresque «Feldkurat » - l'aumônier militaire dont « le brave soldat Chweik » se retrouve l'enfant de chœur - « expédie » au plus court, à l'intention de soldats égayés par ses fantaisies, les messes qui précèdent leur départ au front :

(46) Il remplit brillamment sa tâche comme toujours. Pour la transsubtantiation il se servit cette fois de Weinspritz, et le sermon fut un peu plus long, car un mot sur trois était suivi par un et cætera et un « évidemment".

"Soldats, dit-il, vous partez aujourd'hui pour le front, et cætera. Elevez vos cœurs et cætera vers Dieu, évidemment. Vous ne savez évidemment pas ce que vous allez devenir, et cætera. " Le sermon continuait sur ce ton. Le courant d'et cætera et d'" évidemment » s'arrêtait parfois pour laisser passer des «nom de Dieu » et les noms de tous les saints. [...] La messe fut cependant achevée sans autre scandale, ayant fort diverti les soldats qui y assistaient [J. Hasek, Le brave soldat Chweik, trad. du tchèque, Gallimard, chap. 12, idt].

Le pointage du déjà-dit d'une formule - par opposition à son assimilation au « propre » du dire - peut aussi passer par son déplacement en contexte discor- 
dant $^{71}$, comme dans cette lettre humoristique adressée à un ami par celui à qui il a prêté sa maison et confié son chien :

(47) J'ai le regret de porter à votre connaissance le fait que le nommé Tiburce a renversé et cassé en courant comme un fou, à son habitude, la jolie poterie bleue de l'entrée de la maison [Corresp. privée, 14-08-2002] ;

ou se faire, au plan d'un genre de discours très normé, par sa bascule dans le genre - de RDA - de la parodie : il en est ainsi, par exemple, dans les jubilatoires caricatures d'articles scientifiques de Perec $^{72}$, ruinant le « sérieux » de la pratique des routines (que celle-ci soit automatisée et comme oubliée par des chercheurs chevronnés, et/ou résignés, ou attentivement suivie par un apprenti...) par la mise en scène distanciante de leur exacerbation et d'une discordance d'avec l'objet farfelu du discours.

Que de l'extériorité discursive accède localement à la conscience de l'énonciateur, c'est ce qui rapproche RDA et conformation délibérée d'un dire à un déjà-dit, par opposition au caractère insu de la présence, constitutive, du déjà-dit dans le dire. Mais là où le dire « ne fait qu'un » avec l'ailleurs auquel il se (con)fond, présentant par là, dans sa forme même cet ailleurs auquel il se conjoint, la RDA qui, par son étagement métalangagier, institue quelque chose de son extériorité perçue comme extérieure au discours en train de se faire, en le représentant comme tel, est foncièrement différenciatrice.

L'opposition entre ces deux modes - assimilateur/dissimilateur - de traitement d'un ailleurs discursif perçu par l'énonciateur ne correspond pas à une opposition accord/désaccord de l'énonciateur avec l'ailleurs consciemment accueilli : «faire UN avec » et « représenter comme autre » un certain discours, se situent en deçà des opinions ou sentiments de l'énonciateur à l'égard de ce discours.

L'opération consciente - mais non dite - de « faire un » avec un déjà-dit peut s’accompagner de la plus grande diversité des affects - pleine adhésion, certes, du sujet à ce qu'il assimile, accord avec un rôle, attention appliquée, mais aussi

71 Déplacement « hétérogénéisant " par rapport au fonctionnement homogène des routines « faites siennes » par un dire - cf. l'analyse pertinente de R. Mahrer (2005: 105) remarquant que « certaines unités du discours » étant « associées [...] à des composantes contextuelles » il s'ensuit que « quand tel énoncé est employé dans un contexte auquel il n'est pas associé habituellement (dans un genre auquel il ne correspond pas selon la représentation dudit genre qu'a le co-énonciateur ou provenant d'un énonciateur auquel le co-énonciateur n'aurait pas prêté une telle parole...) il est alors chargé d'une valeur d'hétérogénéité voire d'impertinence [...]. »

72 « Cantatrix sopranica L. - et autres écrits scientifiques », parodique contribution neuro-physiologique - génériquement hypernormée - de G. Perec à la recherche internationale sur les effets du jet de tomates sur les cantatrices... pour le premier texte du recueil (voir chap. 5 note 29, p. 162). 
bien amusement, irritation, résignation... - éprouvés à « conformer » son dire à un prêt-à-dire/écrire ${ }^{73}$. De même, le geste dissimilateur de la RDA est à entendre comme pure distinction de deux discours, mise en rapport qui ouvre sur toute la gamme des rapports possibles entre eux, du conflit à l'accord. La RDA n'est nullement rebelle à l'expression, dans le discours, de son identification avec le dire autre de $\boldsymbol{l}$ que $\mathbf{L}$ partage, qu'il reprend à son compte, auquel il s'associe, qu'il rejoint, etc. Mais il faut souligner la différence entre appropriation, consciente mais implicite, d'un extérieur discursif par un discours, et mise en scène explicite dans un Discours d'un autre discours distinct et du mouvement par lequel il « fait un » avec lui. À titre d'illustration de l'emphase dont peut se charger - loin de l'assimilation muette - la représentation de ne « faire qu'un » avec un autre bien identifié comme tel, je renvoie au florilège des énoncés de RDA émanant de députés abolitionnistes lors du débat parlementaire sur la peine de mort, en 1981, analysés par R. Micheli ${ }^{74}$ comme relevant de «stratégies de rapprochement », tendant à " réduire la distance » d'avec une source prestigieuse, fortement " distinguée », pour en arriver à se « réapproprier » le discours de celle-ci, comme par exemple :

(48) - À ce sujet, je fais miennes les paroles de notre camarade Jean Jaurès, que vous avez si bien rappelées hier, Monsieur le Garde des Sceaux « Je crois pouvoir dire, déclarait-il voici soixante treize ans, que la peine de mort est contraire à ce que l'humanité [...] ».

-Enfin, je terminerai en évoquant celui qui siégea ici (l'orateur montre la place où siégea Victor Hugo) [...] celui qui s'est battu toute sa vie contre la peine de mort et qui, le 15 septembre 1848 prononçait devant l'Assemblée Nationale, cette phrase que je fais mienne : «Je vote l'abolition pure, simple et définitive de la peine de mort. »

ou :

(49) Ce monument ne devait jamais être réalisé, mais il anticipe ce jugement de Chateaubriand que nous faisons nôtre : "Devant le tombeau de Napoléon, on ne perçoit que le génie de Napoléon ; devant celui de Louis XIV on perçoit le génie de la France » [G. de Saint-Bris Louis XIV et le Grand Siècle, 2012, L. de Poche, p. 343].

(50) Enfin, et surtout, je fais mienne la formule de Victor Hugo ; «Il y a dans l'admiration quelque chose de réconfortant. " J'adore admirer... [B. Tavernier, Conclusion d'un entretien dans Télérama, 15/21-10-2016, p. 8, idt].

À l'opposé du processus d'assimilation - relevant d'un « faire sien » tacite ou ignoré - ce mouvement rhétorique de « faire sien » suppose la « dissimilation explicite » de la RDA.

73 Affects aussi divers que ceux avec lesquels on peut être amené à revêtir un uniforme, par opposition avec un déguisement ludique, dans lequel le "sérieux" du "faire un" serait ruiné par des éléments de "distanciation" (tels la discordance avec la situation, l'ostentation, etc., comme, par exemple en (22), (47) ou le texte "scientifique" de Perec).

74 Micheli (2006 : 291 sq.) évoqué ci-dessus au chap. 5.5.4.2, p. 191. 


\section{Entre l'autre et le propre, une zone sensible}

Par le tracé différenciateur - dissimilateur - de la RDA, le Discours partage en lui-même, les territoires des discours autres et ceux de sa parole " propre », foncièrement ou consciemment « faite sienne ». Élément « configurateur » essentiel du discours en train de se faire - ce tracé - métadiscursif - n'a donc pas la netteté tranchante d'une ligne tracée au cordeau : du flou apparaît dans la cartographie sur une marge, une frange de la RDA, où le partage - au sens de séparation - entre ce qui relève de l'un ou de l'autre se fait incertain.

Deux données se combinent, on l'a vu, pour brouiller la ligne de partage que trace, par exemple, un DD maximalement marqué : un fait de langue - les degrés de marquage que présentent tous les modes de RDA, avec la possibilité d'une réalisation en marquage zéro - et un fait de langage - la présence constitutive, qu'elle soit formulée en termes de dialogisme foncier ou d'extériorité interne, de l'ailleurs discursif dans le dire.

À ces formulations théoriques répondent, pour les énonciateurs, l'expérience concrète de cette présence sous-jacente au dire : jouant sur un mode largement et nécessairement ignoré des sujets parlants - puisque parler et comprendre se font dans, à travers, via le déjà-dit qui constitue la " matière » même du dire et du sens - cette présence peut, à tout moment, affleurer à la surface « unie »du dire, de façon plus ou moins prévisible ou inopinée, et plus ou moins vive, à la conscience de l'un, et pas de l'autre, des co-énonciateurs : c'est cette " présence », perçue différemment, interprétée différemment, qui est au cœur des flottements, malentendus, conflits, surprises évoqués ci-dessus.

Au principe de ces discordances ouvertes par le non marqué, tout ce qui relève d'un écart : écart notamment entre les pôles de la co-énonciation, immédiate ou différée, dont les mémoires discursives, foncièrement dissemblables, ne sont, de plus, pas " activées » de façon immuable, écart temporel entre un énonciateur et lui-même en position de récepteur, écart dans le moment même de l'énonciation-réception entre le conscient et l'inconscient du dire. Et, affectant toutes les opérations liées à ces « affleurements » de déjà-dit dans le dire, le dégradé des niveaux de conscience ${ }^{75}$ où elles se situent : conscience aussi affirmée que

75 Gradation soulignée par Bakhtine (1984). Arrivé (2012) fait apparaître combien la question de la conscience que le sujet parlant a de la langue n'est pas étrangère à la réflexion de Saussure (2002), et relève notamment l'attention de celui-ci « aux degrés de conscience » correspondant à l'idée que « la notion de conscience est éminemment relative », et suggère (p. 37, 40) que le métalangage - non désigné par ce mot chez Saussure - correspond chez lui au « degré de conscience le plus élevé » ou à « la forme la plus aigüe de "la conscience de la langue” ». Transporté au plan de la conscience plus ou moins vive, en un point, de la présence d'un extérieur discursif, c'est au 
celle qui accompagne le marquage comme autre d'un élément, mais aussi demiconscience, conscience latente, instable, non-conscience et inconscient ${ }^{76}$. Ainsi la ligne séparatrice par laquelle le discours assigne en lui-même un territoire au discours autre se défait-elle - conflictuelle, instable, incertaine jusqu'à l'indécidable, « poreuse »... en une zone indécise entre l'un et l'autre.

Pour autant, ce n'est nullement une sorte de zone «neutre » où s'émousserait la « question » de la frontière : loin d'être mise en sommeil faute de repères assurés, elle est au contraire avivée par l'incertitude. La vivacité des réactions des co-énonciateurs - conflits, malaises, irritation, surprise... comme l'intensité des quêtes de repères manquants, font apparaître ce tracé comme d'autant plus « sensible » qu'il est incertain, témoignant par là des enjeux qui y sont attachés, pour le sens et pour le sujet énonciateur. L'inconfort, le trouble que font naître, ponctuellement, dans le dire, les flottements et incidents de frontières qui s'y produisent, apparaissent ainsi comme des révélateurs de ce qui, «sans histoire » est normalement assuré par la RDA : la séparation configurante de l'un d'avec l'autre par laquelle, dans l'imaginaire ${ }^{77}$, pour le discours et son sujet-énonciateur se dessine une identité au reflet différenciant de « ses » autres (qu'on évoquera chap. 12) et se conforte le sentiment d'une parole « propre » (chap. 13, 14).

degré maximal, en effet, qu'on pourrait placer le marquage métadiscursif d'une MAE; voir aussi Arrivé (2018)..

76 Ce qui, rappelons-le, n'est aucunement synonyme. Le non-conscient relève d'une absence, là où l'inconscient est présence agissante ; $c f$. ex. (41) et (42) où l'ailleurs, chargé de sens est positivement mis en jeu par l'inconscient.

77 Cette catégorie - on l'a vu ci-dessus chap. 10.3.2, p. 410 - ne renvoie pas au monde des fées et des licornes, mais à la part d'illusion nécessaire du « Moi » sans laquelle un sujet humain ne peut « tenir». 